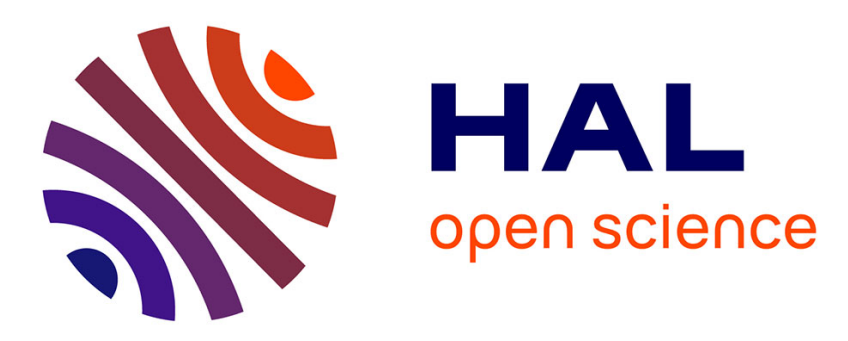

\title{
Functional Analysis of the Sensory Motor Pathway of Resistance Reflex in Crayfish. II. Integration of Sensory Inputs in Motor Neurons
}

Didier Le Ray, François Clarac, Daniel Cattaert

\section{- To cite this version:}

Didier Le Ray, François Clarac, Daniel Cattaert. Functional Analysis of the Sensory Motor Pathway of Resistance Reflex in Crayfish. II. Integration of Sensory Inputs in Motor Neurons. Journal of Neurophysiology, 1997, 10.1152/jn.1997.78.6.3144 . hal-02346675

\section{HAL Id: hal-02346675 \\ https://hal.science/hal-02346675}

Submitted on 7 Nov 2019

HAL is a multi-disciplinary open access archive for the deposit and dissemination of scientific research documents, whether they are published or not. The documents may come from teaching and research institutions in France or abroad, or from public or private research centers.
L'archive ouverte pluridisciplinaire HAL, est destinée au dépôt et à la diffusion de documents scientifiques de niveau recherche, publiés ou non, émanant des établissements d'enseignement et de recherche français ou étrangers, des laboratoires publics ou privés. 
FUNCTIONAL ANALYSIS OF THE SENSORY MOTOR PATHWAY OF RESISTANCE REFLEX IN CRAYFISH. II- INTEGRATION OF SENSORY INPUTS IN MOTOR NEURONS.

Didier LE RAY, François CLARAC, and Daniel CATTAERT

Laboratoire de Neurobiologie et Mouvements, CNRS, 31 chemin Joseph Aiguier, Marseille, France.

Running title: Sensory integration in identified synapses.

Correspondence: D. LE RAY, Laboratoire Neurobiologie et Mouvements, CNRS, 31 chemin Joseph Aiguier, 13402 Marseille cedex 20, FRANCE.

Tel: (33) 4. 91.16.43.45

Fax: (33) 4. 91.77.50.84

E-mail: leray@1nf.cnrs-mrs.fr 


\section{ABSTRACT}

The in vitro preparation of the fifth thoracic ganglion of the crayfish was used to analyze the wiring of the monosynaptic reflex responses recorded from the depressor motor neurons (Dep MNs). Dep MNs are directly connected by the release-sensitive afferents issued from a proprioceptor, the coxo-basipodite chordotonal organ ( $\mathrm{CBCO})$ which is released by upward movements of the leg. Sine wave movements, applied to the CBCO strand from the most released position, allowed us to stimulate the greatest part of release-sensitive CBCO fibers. Systematic intracellular recordings from all Dep MNs performed in high divalent cation saline allowed us to determine the connections between $\mathrm{CBCO}$ afferents and their postsynaptic Dep MNs: it highlighted the sequential activation of the different Dep MNs involved in the monosynaptic reflex activities. The study of the convergence of sensory inputs onto a given Dep MN, and the study of the divergence of a given sensory input onto several Dep MNs pointed out the complexity of the sensory-motor reflex loops involved in the control of locomotion and posture. Electrophysiological experiments and simulations were performed to analyze the mechanisms by which Dep MNs integrate this great amount of sensory inputs. Paired intracellular recording experiments demonstrated that both postsynaptic response shapes could be induced by varying the presynaptic firing frequency, whatever the postsynaptic Dep MN. Compartment model simulations were used to analyze the role of the sensory-motor synapse characteristics in the summation properties of postsynaptic MN. Distal and proximal synapse localizations were tested upon models with various membrane resistivities (that allowed us to range the possible values observed in MNs): simulations demonstrated the substantial role of the postsynaptic compartment geometry, since large postsynaptic compartments allowed to generate greater EPSP summations than small ones. Functional implications of the wiring of the resistance reflex are discussed. 


\section{INTRODUCTION}

The role of sensory information, especially proprioceptive feedback (Sherrington 1910), in the organization of motor output has been studied extensively in a variety of different preparations, invertebrate and vertebrate alike (Barnes and Gladden 1985). Proprioceptive inputs may be integrated at different levels of the motor command. The simplest interactions consist of direct contacts between proprioceptive sensory afferents and motor neurons (MNs). More complex integrations involve one or several interneurons. For example, in the cat spinal cord, Ia afferents evoke monosynaptic excitatory postsynaptic potentials (EPSPs) and Ib afferents evoke disynaptic inhibitory postsynaptic potentials (IPSPs) in homonymous MNs (Eccles 1964). Polysynaptic connections also exist both in insect (Bässler 1993; Burrows and Pflügers 1988; Büschges and Wolf 1995) and in crustacea (Clarac et al. 1991; Le Ray and Cattaert 1997). Recent intracellular studies have shown that simple neuronal network activity depends upon connectivity, and endogenous properties of component neurons (Harris-Warrick et al. 1992). Therefore sensory-motor integration may imply more complex dynamic processes.

However, studies of sensory-motor interactions mainly concern simple reflexes. Among these, a great amount of data concerns the vertebrate stretch reflex: it is a negative feedback loop in which stretching the muscle spindle evokes activation of homonymous MNs (Eccles 1957; Granit 1955; Matthews 1972). For the last 40 years, physiological studies of this reflex have demonstrated that most of the excitatory connections between sensory afferents and MNs are monosynaptic (Burke and Rudomin 1977; Redman 1979). In arthropods, the monosynaptic connections between primary afferents from proprioceptors and MNs is a widespread phenomenon too. In all cases, these connections support resistance reflexes, comparable to the vertebrate stretch reflex, in which MNs respond to counteract the imposed 
movement. In insect, the monosynaptic connections have been demonstrated for the femoral chordotonal organs of the locust (Burrows 1987). In the crustacean walking leg, Blight and Llinás (1980) described monosynaptic connections in the thoraco-coxal muscle receptor organ (TCMRO). More recently, in the crayfish walking legs, afferents from the coxo-basipodite chordotonal organ $(\mathrm{CBCO})$ were demonstrated to make direct excitatory synapses onto levator (Lev) and depressor (Dep) MNs: stretch-sensitive sensory fibers connect Lev MNs and release-sensitive fibers connect Dep MNs (El Manira et al. 1991).

Although systematic analysis of the coding of movement parameters have been extensively performed (Bush 1965a, b; Le Ray et al. 1997; Matheson 1990), the organization of the reflex responses, and particularly the role of a given sensory information in this organization, remains unclear. The sensory-motor loop involving the $\mathrm{CBCO}$ in the crayfish thoracic in vitro preparation gives us the possibility to unravel this question: only around 20 sensory afferents connect less than ten Dep MNs (Le Ray and Cattaert 1997). In an associated work, we performed the complete classification of the $\mathrm{CBCO}$ release-sensitive fibers and of the Dep MN reflex responses (Le Ray et al. 1997).

In this paper, we have analyzed what type of information each identified Dep MN received from the $\mathrm{CBCO}$ during imposed movements. A functional wiring diagram is established, and the role of EPSP characteristics in the integration of the MN response is analyzed using a compartment model simulation.

\section{METHODS}

\section{Preparation.}


Results are based on > 130 intracellular recordings from CBTs and Dep MNs that were performed on adult male and female crayfish, Pacifastacus leniusculus and Procambarus clarkii. Animals were maintained in aquarium at $18^{\circ} \mathrm{C}$ and fed once a week.

The in vitro preparation consisted in the last three thoracic ganglia and the two couples of antagonistic motor nerves innervating the two proximal joints of the $5^{\text {th }}$ leg (Promotor/Remotor and Depressor/Levators). The CBCO, which encodes the vertical movements of the leg, was dissected out together with its sensory nerve. The preparation was pinned down dorsal side up in a Sylgard-covered Petri dish and superfused with oxygenated crayfish saline.

\section{Stimulations / Recordings.}

Extracellular recordings were performed using pin platinum electrodes contacting the nerves, isolated from bath with petroleum jelly (Vaseline), and directed to a 4-channel differential AC amplifier (A-M Systems). Single and paired intracellular recordings from CBTs and Dep MNs (Fig. 1, A and B) were realized with thin-walled glass micro-electrodes filled with a potassium chloride solution (3M) and having a $25-$ to $30-\mathrm{M} \Omega$ resistance. The signals were amplified by an Axoclamp 2B (Axon Instruments). Intracellular current pulses were controlled by an 8-channel digital stimulator (A.M.P.I.). All signals were monitored on a eight channel oscilloscope, a four channel digital oscilloscope (Yokogawa DL 1200) and stored on D.A.T. tapes (BioLogics digital tape recorder) and digitized on a PC-based computer through an A/D interface (from Cambridge Electronic Device, CED 1401PLUS). Intracellular and extracellular recordings were digitized at $20 \mathrm{kHz}$ and written to disk.

A homemade puller controlled mechanical stimuli characterized by cyclic stretch and release of the CBCO strand, according to a sinusoidal (Fig. 1C) or a ramp protocol (Fig. 4). Movement stimulations were performed from the most released position of the CBCO strand, and total movement amplitude was one third of the released $\mathrm{CBCO}$ strand length 
(1 mm - $1.8 \mathrm{~mm})$. The movement control voltage traces were visualized on the oscilloscopes and stored on both tape and computer.

\section{Salines.}

Vaseline wall was used to superfuse separately the $\mathrm{CBCO}$ and the thoracic ganglia. The $\mathrm{CBCO}$ was superfused with saline which contains (in $\mathrm{mM}) 195 \mathrm{NaCl}, 5 \mathrm{KCl}, 13 \mathrm{CaCl}_{2}, 2$ $\mathrm{MgCl}_{2}$. The thoracic ganglia was superfused with saline where divalent cation concentration was increased $\left[(\right.$ in $\mathrm{mM}) 34 \mathrm{CaCl}_{2}, 6.4 \mathrm{MgCl}_{2}$, with the sodium concentration reduced accordingly], in order to raise the activation threshold of all central neurons without affecting the CBCO sensory inputs. It allowed to unmask the monosynaptic reflex response from MNs (Berry and Pentreath 1976). Moreover, in experiments where intracellular current pulses were delivered into the CBTs, the high divalent cation concentration saline did not affect the monosynaptic EPSP produced in the post-synaptic Dep MN (Fig. 5B). Saline solutions were buffered with $3 \mathrm{mM} \mathrm{N}$-2-hydroxyethylpiperazine-N'-2-ethanesulfonic acid (HEPES) and pH adjusted at 7.7 at $15^{\circ} \mathrm{C}$.

Analysis.

Physiological signals were analyzed with the CED SPIKE2 program. Data and traces were acquired using scripts running with the SPIKE2 program. The SPIKE2 "Wave Marker" tool allowed to identify extracellular CBCO units from the neurogram. Subsequently, it was possible to determine which sensory unit connected a given intracellularly recorded assistance (A MN) or resistance (R MN1 and 2) Dep MN (Fig. 1C, 1 and 2).

Circular statistics were used to analyze the distribution of identified CBCO EPSPs recorded from a given $\mathrm{MN}$ during imposed sine wave movements. The phase occurrence of each sensory unit over several movement cycles (starting of the release phase being chosen as zero) was figured by a unit vector $\vec{v}_{i}$. The resulting vector $\vec{R}$ was calculated as the sum of all (n) unit vectors $\vec{v}_{i}$ divided by n (Fig. 1, C3, left), for each identified CBCO unit: 


$$
\begin{gathered}
\vec{R}=\frac{\sum_{n} \vec{v}_{i}}{n}, \\
\|\vec{R}\|=\sqrt{R_{x}{ }^{2}+R_{y}{ }^{2}}, \\
\alpha=\operatorname{ArcTan}\left(\frac{R_{y}}{R_{x}}\right),
\end{gathered}
$$

with $\mathrm{R}_{\mathrm{x}}$ and $\mathrm{R}_{\mathrm{y}}$ representing the $\mathrm{x}$ and y coordinates of the resulting vector $\vec{R}$. The resulting vector length $(\|\vec{R}\|)$ has a value between 0 and 1 , and is a measure for the mean distribution of spikes within a stimulus cycle: a value of zero would indicate that the spikes were evenly distributed; a value of one would indicate that all spikes occurred at the same phase. In Fig. 1-C3, the right circular diagram illustrates distribution of the resulting vectors of the three different CBCO units (1, 2 and 4) that make direct EPSP in R MN1 (illustrated in Fig. 1-C2). Statistical analysis and fittings were performed with the GraphPad Prism statistic programs.

\section{Simulations.}

Spatial and temporal integration of EPSPs produced at different locations of MN dendrites, was simulated with a compartment model (program SWIM, Ekeberg et al. 1991). The model allows construction of arbitrarily complex dendritic trees. The simulation model used 60 compartments. The main neurite, from which intracellular recordings are usually performed in real MNs, was composed of 20 compartments each $20 \mu \mathrm{m}$ long and $10 \mu \mathrm{m}$ in diameter. In addition, two primary branches, one composed of 15 compartments (length: 20 $\mu \mathrm{m}$; diameter: $2 \mu \mathrm{m}$ ) the other composed of 7 compartments (length: $20 \mu \mathrm{m}$; diameter: 10 $\mu \mathrm{m}$ ), and two secondary branches composed of 8 compartments (length: $20 \mu \mathrm{m}$; diameter: $2 \mu \mathrm{m})$ were simulated too. In real crayfish MNs, input synapses are likely to be located on primary and secondary neurite branches. Because no active spike propagation occurs in crayfish MN neurites, only passive electrical properties were simulated: 
The intra-compartment potential $\mathrm{E}$ is described by the differential equation

$$
\frac{d E}{d t}=\frac{I_{\text {leak }}+I_{\text {core }}+I_{c h}+I_{s y n}}{c m} \text { (equation 1) }
$$

$I_{\text {leak }}$ models passive leakage through the cell membrane. It is

$$
I_{\text {leak }}=\left(E_{\text {leak }}-E\right) \cdot G_{\text {leak }}
$$

where the parameters $E_{\text {leak }}$ and $G_{\text {leak }}$ are the equilibrium potential and the leak conductance respectively. $I_{\text {core }}$ is the electrical coupling to neighboring compartments summed over all neighbors

$$
I_{\text {core }}=\sum_{c \in \text { neighbors }}\left(E_{c}-E\right) \cdot G_{\text {core }}
$$

The parameter $G_{\text {core }}$ denotes the core conductance from the compartment in question to the neighboring compartment.

$I_{\mathrm{ch}}$ and $I_{\mathrm{syn}}$, in equation 1 , model the flow of ions through active channels and synapses respectively. The parameter $\mathrm{cm}$ describes the capacity of the compartment

$$
\mathrm{cm}=C_{m} . \text { area }
$$

(area is the membrane surface of the compartment, $C_{\mathrm{m}}$ is the specific capacitance).

All compartments had the same membrane resistivity $R_{\mathrm{m}}$ that was adjusted to 4000 or 8000 $\Omega . \mathrm{cm}^{2}$. All computations were carried out assuming a specific capacitance $C_{\mathrm{m}}$ of $1 \mu \mathrm{F} / \mathrm{cm}^{2}$ and a cytoplasmic resistivity $R_{\mathrm{i}}$ of $75 \Omega . \mathrm{cm}$.

Synaptic excitatory input was modeled with a conductance in the postsynaptic compartment, and an activation level of the postsynaptic channel, $s$, which is 0 if the synapse is "closed" and 1 if it is fully "opened". The maximum conductance $G_{\text {syn }}$ is fixed, but the value of the actual conductance $G_{\mathrm{syn}} s$ varies with $s$; the kinetic of $s$ is controlled by two parameters: the duration and the decay time. The synaptically induced current that enters the postsynaptic compartment is calculated by 


$$
I_{\text {syn }}=\sum_{\text {synapses }}\left(E_{s y n}-E\right) \cdot G_{s y n} S
$$

in which $E_{\mathrm{syn}}$ is the equilibrium potential for EPSPs.

\section{RESULTS}

In a previous work, Le Ray and Cattaert (1997) described the Dep MN monosynaptic reflexes elicited by ramp movements imposed to the $\mathrm{CBCO}$ strand. They demonstrated the existence of three types of Dep MN response: Three Dep MNs did not present any monosynaptic response, one displayed an assistance reflex response (i.e. depolarizations during the stretch of the $\mathrm{CBCO}$ ), and eight Dep MNs produced a resistance response (i.e. depolarizations during the $\mathrm{CBCO}$ release). Moreover, in an associated work, we did an extensive analysis of the release sensory coding, and demonstrated the existence of two distinct resistance responses: one characterized only by phasic EPSP bursts related to release ramps; the other characterized by both phasic bursts of EPSPs and a graded membrane depolarization during the release phase of the movement (Le Ray et al. 1997). The present study aimed at analyzing the transfer of information from $\mathrm{CBCO}$ fibers to monosynaptic output Dep MNs.

I- Projections of CBCO units onto the Dep MNs - Extracellular and intracellular studies.

In order to find out the type and number of $\mathrm{CBCO}$ afferents that project onto a given MN, we performed systematic intracellular recordings from all Dep MNs in single 
experiments, and used the procedure described in figure $1 \mathrm{C}$ to identify the different $\mathrm{CBCO}$ units from the sensory nerve. Figure 2 shows the results obtained in each of the nine Dep MNs that received monosynaptic $\mathrm{CBCO}$ inputs, impaled successively in the same experiment. The figure presents the resulting vector of each of the $\mathrm{CBCO}$ afferents that connect the MNs (thin lines). From three to seven (mean $=5$ ) afferents contacted each resistance Dep MN, and four afferents contacted the assistance Dep MN. This representation confirmed that some resistance Dep MNs received monosynaptic information even during the stretch of the CBCO. In order to represent the activity of all the $\mathrm{CBCO}$ afferents that connect a given $\mathrm{MN}$, the corresponding resulting vectors were averaged (thick line, out of each circle). Although five resistance Dep MNs received one stretch-sensitive input, it was obvious that the greatest number of $\mathrm{CBCO}$ inputs were received during the $\mathrm{CBCO}$ release (white part of the cycle). The sectors of imposed movement in which Dep MNs received the greatest number of monosynaptic EPSPs are represented on the right of each circular diagrams (dark part of the sine waves). It appeared that not all the resistance Dep MNs received the same information concerning the imposed movement and the position of the leg. It seems that each of the resistance Dep MNs was excited preferentially during a limited part of the movement. The same kind of temporal organization has been found in all these experiments $(n=4)$.

Table 1 summarizes the results obtained in several experiments. In our conditions, the assistance Dep MNs received about five exclusively stretch-sensitive sensory units. The resistance Dep MNs received an averaged number of six CBCO units (from three to eight) in sine wave movement conditions. Resistance Dep MNs seemed to receive inputs from a larger variety of $\mathrm{CBCO}$ units than the assistance Dep MN: The organization of CBCO afferent projections onto the Dep MNs is given in Table 1. Although all of the impaled resistance Dep MNs mostly received release-sensitive CBTs, some (17 Dep MNs) received also inputs from 
non-specific $\mathrm{CBCO}$ units, and in some cases (four Dep MNs) from stretch-sensitive CBCO units.

In some experiments, we determined the number of postsynaptic Dep MNs that received monosynaptic EPSPs from a given sensory unit. We focused exclusively upon resistance Dep MNs, using both extracellular and intracellular approaches. Figures 3 and 4 show two examples of the results obtained with both methods. In experiments where sinusoidal movements were applied to the $\mathrm{CBCO}$ strand, we impaled successively the different postsynaptic Dep MNs and extracellularly recorded the CBCO units. Figure 3 presents one of these experiments in which 14 different $\mathrm{CBCO}$ units were identified and the eight postsynaptic resistance Dep MNs were impaled two by two successively. Most of the recorded CBCO units elicited EPSPs in only a few number of postsynaptic Dep MNs: ten of the sensory afferents do not project upon more than one or two different Dep MNs. Nevertheless, some CBCO units elicit monosynaptic EPSPs in several resistance Dep MNs (from three to eight distinct postsynaptic Dep MNs); there exists one $\mathrm{CBCO}$ afferent that produce EPSPs in each of the eight resistance Dep MNs. It is noticeable that the same CBCO afferent is able to produce different shape and amplitude EPSPs in the different postsynaptic Dep MNs (see the averaged EPSPs in Fig. 3). Thus, there exist both a divergence and a convergence of the sensory informations onto the different Dep MNs.

In some experiments, ramp movements were applied to the $\mathrm{CBCO}$ strand and successive paired intracellular recordings were performed from a given CBT and several resistance Dep MNs. In figure 4, while a phasico-tonic release-sensitive CBT was intracellularly recorded, we impaled successively all 12 Dep MNs. The phasico-tonic releasesensitive CBT connected monosynaptically five different resistance Dep MNs (distinguished with their different extracellular spikes), and produced EPSPs of different shape and size (see 
averaged EPSPs in the right part of Fig. 3). These results confirmed the divergence of one sensory information onto different Dep MNs.

II- "Manipulation" of the signal transmission.

In order to confirm that the $\mathrm{MN}$ response to ramp stimulation of the $\mathrm{CBCO}$ strand was due exclusively to the temporal characteristics of the sensory inflow (since the associated paper demonstrated that MN properties were not involved), we analyzed the effect of a single sensory input onto the MN reflex response. We therefore performed paired intracellular recordings from both a CBT and a Dep MN. Injection of depolarizing current pulses at high frequency rates (30 to $200 \mathrm{~Hz}$ ) into the CBT were then used to study the properties of sensorymotor synapses in high $\mathrm{Ca}^{2+}$ and high $\mathrm{Mg}^{2+}$ saline. In such experiments, high frequency stimulations evoked sustained depolarizations in Dep MNs (Fig. 5A), and each presynaptic spike was capable of producing an EPSP in the postsynaptic Dep MN (Fig. 5B). As presented in figure 3, CBCO-related EPSPs displayed different time constants. The graph in figure 5C presents the plot versus time of the relative exponential decay of repolarizing phase from six representative EPSPs. Each one-phase exponential decay fitting allowed us to calculate the time constant $(\tau)$ for each CBCO-related EPSP: the extreme $\tau$ values ranged from $2.5 \mathrm{~ms}$ up to $12.5 \mathrm{~ms}$, but most of the Dep MN EPSPs had a $\tau$ value between 6 and $7 \mathrm{~ms}$.

Modification of the Dep MN responses by controlling the CBT firings.

An associated paper showed the existence of several kinds of CBCO unit firings and two types of resistance reflex responses in Dep MNs (Le Ray et al. 1997). By using various 
combinations of stimulation frequencies, it was possible to mimic the different CBT activities recorded during ramp movements and, consequently, to analyze the different Dep MN monosynaptic responses (results presented from six experiments). Figures 6 and 7 present the normal and modified reflex responses of both phasic and phasico-tonic Dep MNs in high $\mathrm{Ca}^{2+}$ and high $\mathrm{Mg}^{2+}$ saline.

Figure 6A shows the normal resistance reflex of a phasic Dep MN in response to the application of a ramp stimulation to the $\mathrm{CBCO}$ strand (only the release phase of the stimulation is presented). This Dep MN reflex response was characterized by bursts of EPSPs occurring only during the release ramps. When the CBCO stimulation was stopped (Fig. 6B), it was possible to reproduce a sensory phasic firing by intracellular injection of depolarizing current pulses $(+8 \mathrm{nA}, 3 \mathrm{~ms}, 40 \mathrm{~Hz})$ delivered in trains (0.2-s duration, 1-s interval) into a presynaptic CBT. In these conditions, the reflex response of the post-synaptic Dep MN was comparable (although the EPSP amplitudes were lower) to its normal resistance response (Fig. 6B1). Another CBT intracellular stimulation protocol (+8 nA, [40 Hz, $0.5 \mathrm{~s}],[20 \mathrm{~Hz}, 2$ s], [40 Hz, $0.5 \mathrm{~s}],[30 \mathrm{~Hz}, 2 \mathrm{~s}],[40 \mathrm{~Hz}, 0.5 \mathrm{~s}]$ ) allowed us to transform the same CBT firing in the way to reproduce a phasico-tonic pattern (Fig. 6B2). In these conditions, the post-synaptic Dep MN response resembled the phasico-tonic type of reflex response, i.e. the membrane potential did not return to its resting value between each $40-\mathrm{Hz}$ bursts, but was gradually depolarized in relation with the CBT tonic frequency. Thus, the control of the firing of only one presynaptic CBT was sufficient to transform the Dep MN phasic reflex response into a phasico-tonic-like reflex response.

Figure 7A shows the normal resistance reflex of a phasico-tonic Dep MN in response to the application of a ramp stimulation to the CBCO strand (only the release phase of the stimulation is presented). In the absence of movement (Fig. 7B), in order to reproduce a phasico-tonic sensory firing, we injected depolarizing current pulses $(+12 \mathrm{nA},[100 \mathrm{~Hz}, 0.5 \mathrm{~s}]$, 
[50 Hz, $2 \mathrm{~s}],[100 \mathrm{~Hz}, 0.5 \mathrm{~s}],[80 \mathrm{~Hz}, 2 \mathrm{~s}]$ ) in a presynaptic CBT. The evoked Dep MN response resembled the physiologic one, i.e. great phasic EPSP bursts added onto a graded tonic depolarization of the membrane (Fig. 7B1). The firing pattern of the single presynaptic CBT was modified in order to reproduce a phasic CBT discharge (Fig. 7B2) by intracellular injection of depolarizing current pulses $(+12 \mathrm{nA}, 3 \mathrm{~ms}, 100 \mathrm{~Hz})$ delivered in trains $(0.5-\mathrm{s}$ duration, 2-s interval). Consequently, the postsynaptic Dep MN response was transformed in a purely phasic reflex response.

High frequency EPSP summations in phasic and phasico-tonic Dep MNs.

In paired intracellular recording experiments $(n=9)$, we compared the different postsynaptic Dep MN responses produced by high-frequency stimulation of a single CBT. During high frequency CBT stimulations, summation of EPSPs evoked in the Dep MN resulted in a sustained depolarization (Fig. 5A). This effect was progressive, the rate of depolarization depended upon the stimulation frequency and the stimulated CBT. Graphs representing the rates of depolarization for both phasic and phasico-tonic Dep MNs are shown in figure 8 .

The responses obtained in a phasic Dep MN with the same CBT for 50 and $200 \mathrm{~Hz}$ stimulations are presented in figure $8 \mathrm{~A}$ (top). The maximum depolarization amplitude was four time higher and was reached four time faster for $200 \mathrm{~Hz}$ stimulation frequency than for $50 \mathrm{~Hz}$ stimulation frequency. Therefore, the rate of EPSP summation was strongly frequencydependent for that CBT. However, great differences were observed among the CBTs that connect a single Dep MN, since the same stimulation frequency $(200 \mathrm{~Hz}$ ) could produce motoneuronal responses of quite different amplitudes (one to four folds, Fig. 8A bottom). 
Nevertheless, each of these CBTs evoked frequency-dependent motoneuronal response comparable to that shown in figure 8A (top).

Similar results were found with a postsynaptic phasico-tonic Dep MN. The amplitude of the depolarization was frequency-dependent (Fig. 8B, top). The maximum depolarization amplitude was three time higher for $200 \mathrm{~Hz}$ stimulation frequency than for $50 \mathrm{~Hz}$ stimulation frequency, but was reached nearly at the same time $(50 \mathrm{~ms})$. Here again, great differences were observed among the CBTs that connect a single Dep MN, because the same stimulation frequency $(100 \mathrm{~Hz})$ could produce motoneuronal responses of quite different amplitudes (one to three folds, Fig. 8B bottom).

III- Simulation of the sensory-motor synapse.

Simulation experiments were performed to analyze the integration processes of the afferent signal in the postsynaptic MN. The simulated $\mathrm{MN}$ is restricted to a main neurite, two primary and two secondary branches in which the conduction is passive. The input synapses can be located in different parts of the postsynaptic MN (to match more to reality, synapses were only located on primary and secondary branches). Then, it is possible to simulate the spatial and temporal integration of one or several sensory inputs.

A first set of simulations was performed in order to analyze the role of spatial arrangement of synapses, and of geometry of the postsynaptic compartments in the shape of the centrally recorded EPSP (Fig. 9). One possibility could be that the shape of the EPSP is the same (possibly with various amplitude) whatever the compartment in which it is produced, but because of passive propagation in dendrites, its temporal characteristics change and could give rise to the whole range of EPSP shapes recorded from the main neurite (see figure 3). 
Moreover, during a high frequency train of EPSPs, the amplitude, time to peak and duration of each EPSP should be responsible for dissimilarities in the amount of depolarization (Fig. 8). The shape of EPSPs depends upon the amount of membrane that has to be charged in the postsynaptic compartment. Therefore, we simulated large (20 $\mu \mathrm{m}$ in diameter) and small $(1 \mu \mathrm{m}$ in diameter) postsynaptic compartments (Fig. 9A). On the other hand, the amplitude of EPSPs recorded in the main neurite depends upon the diameter of compartments that are in between the synapse and the recording electrode. In order to maximize the low filtering effects of passive propagation in dendrites, compared to reality, $1 \mu \mathrm{m}$-diameter and $300 \mu \mathrm{m}$-length branches were used. Because membrane resistivity $\left(R_{\mathrm{m}}\right)$ is a parameter difficult to measure in real dendrites, four $R_{\mathrm{m}}$ values were tested $\left(4000,8000,12000\right.$ and $\left.20000 \Omega . \mathrm{cm}^{2}\right)$ in both large and small postsynaptic compartment configurations. The results are presented in graphs of figure 9B: in each case, measurements were made in the main neurite. Peak amplitude, time to peak and time constant of the propagated EPSP were increased with increasing $R_{\mathrm{m}}$. Simulated synaptic inputs in large postsynaptic compartments (filled squares) induced EPSP of smaller amplitude, longer time to peak and larger time constant than in small postsynaptic compartments (filled circles). By comparison, between both postsynaptic compartment configurations, more proximally generated EPSPs (unfilled symbols) did not show as marked differences as distally generated EPSPs (filled symbols). Moreover, proximally generated EPSP presented shorter time to peak and smaller time constant than distally generated one.

The parameters involved in the evolution of trains of EPSPs were studied using the same models. We studied effects of $R_{\mathrm{m}}$ values of $4,8,12$ and $20 \mathrm{k} \Omega . \mathrm{cm}^{2}$, but figure 10 only presents the results obtained for $R_{\mathrm{m}}$ values of $4 \mathrm{k} \Omega . \mathrm{cm}^{2}$ (right) and $20 \mathrm{k} \Omega . \mathrm{cm}^{2}$ (left), for clarity. In order to compare in each case the evolution of membrane potential during EPSP trains, all single simulated EPSPs (distal and proximal) were normalized to the same amplitude (100\%). Three presynaptic spike frequencies $(50,80$ and $140 \mathrm{~Hz})$ were tested on 
each postsynaptic configuration at distal and proximal synapse locations. For both $R_{\mathrm{m}}$ examples, fittings of summations are presented on graphs for $50 \mathrm{~Hz} 80 \mathrm{~Hz}$ and $140 \mathrm{~Hz}$ stimulation frequencies (left: distal synapses; right: proximal synapse); the corresponding simulated raw data are presented for 50 and $140 \mathrm{~Hz}$ stimulation frequencies (left: distal; right: proximal). With increasing frequencies, the depolarization amplitude increased and the rise time of the depolarization decreased. In each case, the distal synapse-induced EPSP summation was always of greater amplitude than the proximal one. Moreover in the large postsynaptic compartment configuration $(20 \mu \mathrm{m}$ in diameter, Fig. $10 \mathrm{~B}$, D), differences between proximally and distally generated compound EPSPs were more marked than in the small postsynaptic compartment configuration $(1 \mu \mathrm{m}$ in diameter, Fig. $10 \mathrm{~A}, \mathrm{C})$. The proximally generated compound EPSP changed very little (less than $1 \%$ of the normalized single EPSP) from one configuration to the other; the main difference resulted in the distally generated compound EPSP which amplitude varied from $2 \%$ (when $R_{\mathrm{m}}$ was $4 \mathrm{k} \Omega . \mathrm{cm}^{2}$ ) to more than $50 \%$ (when $R_{\mathrm{m}}$ was $20 \mathrm{k} \Omega . \mathrm{cm}^{2}$ ) of the normalized single EPSP.

Although the geometry of the postsynaptic neuron seems to be able to determine the shape of the response recorded from the main neurite, the weak variability observed between the two extreme postsynaptic compartment configurations are unable to explain the great differences observed among the EPSPs that can be recorded from real MNs (see figure 3), or observed among the Dep MN reflex responses elicited by CBCO release (see Le Ray et al. 1997). Some other simulations were performed to study the importance of the synapse temporal characteristics. It appeared that long lasting synapses, i.e. synapses with a long decay time, produced EPSPs with long time constant that were able to much more summate than short time constant EPSPs (not shown). Although these EPSPs with long time constant are rarer than short ones (see graph in Fig. 5C),. it seems that properties of synapses between 
sensory afferents and $\mathrm{MN}$ are also determinant for the development of phasico-tonic reflex responses in some Dep MNs.

\section{DISCUSSION}

\section{In vitro and in vivo CBCO strand movements.}

In this study, imposed movements corresponded to the angular range covered during locomotion. We could argue that more $\mathrm{CBCO}$ afferents normally project onto MNs, especially during extreme downward movements of the leg. Consequently, some MNs classified as nonresponding would perhaps respond to these larger movements. However this is unlikely because electrical stimulation of the $\mathrm{CBCO}$ nerve failed to activate monosynaptically these silent MNs (data not shown). Anatomical studies have demonstrated that the CBCO nerve contains 38-41 axonal profiles (diameter ranging from $1 \mu \mathrm{m}$ to $17 \mu \mathrm{m}$ ) (Cattaert, unpublished observations), likely equally divided into release and stretch-sensitive fibers. During release movements, up to 14 distinct release-sensitive $\mathrm{CBCO}$ afferents could be identified from the CBCO neurogram (Fig. 3). Therefore, there exist around six release-sensitive CBCO fibers that were not identified during sine wave movements. Possibly, these units could be activated by release movement starting from extreme stretched positions, and thus not involved during locomotion or posture. Another possibility is that these units were activated by the imposed movements but could not be identified due to their identical shapes in the CBCO neurogram. Nevertheless, if this is the case, the results presented in figure 2 would have been roughly the same (although there would exist more sensory units, the resulting vectors would remain identical). Therefore, this study is very representative of the connections involved in the monosynaptic resistance reflex pathways, during walking or resting activities in crayfish. 
Functional consequences of sensory-motor wiring.

Contrary to the cat spinal cord, in which Ia afferent fibers have been demonstrated to project onto most (65 to 80\%) of the homonymous MNs (Nelson and Mendell 1978; Scott and Mendell 1976), in crayfish half of the release-sensitive CBCO fibers project onto only one Dep MN (Fig. 3). This would indicate some specific input-output connections. However, this does not seem to be the case since each Dep MN receives several (from two to five) CBCO afferents coding different angular sectors, and therefore integrates a compound sensory signal. However, none of the MNs realizes the same proprioceptive integration. These integration processes result in the different Dep MNs being sequentially activated during passive levation of the leg (Fig. 2). By comparison of the results obtained in other preparations, convergence of proprioceptive inputs is a widespread phenomenon also described in the crayfish terminal abdominal ganglion (Newland and Nagayama 1993) as well as in the stick insect (Sauer et al. 1996) as in the locust (Burrows 1987) and as in the leech (Lockery and Kristan 1990). However, in these cases, proprioceptive afferents mainly project onto spiking or non-spiking interneurons which decide of the motor response (shape, strength and direction). Surprisingly, convergence of direct connections from proprioceptive afferents onto MNs are a shared feature of resistance reflex in crayfish and stretch reflex in vertebrates (Eccles et al. 1957). This common characteristic would lead to a very rigid reflex behavior that is avoided by the mean of presynaptic inhibition of central origin in both cases (Cattaert et al. 1992; Eccles et al. 1962).

In opposition to the convergence of CBCO inputs onto resistance Dep MNs (about five CBCO units per MN), divergence is quite heterogeneous (from one to eight MNs connected by a single $\mathrm{CBCO}$ fiber; see figure 3 ). This characteristic would allow differential regulation of the sensory-motor connections: presynaptic inhibition of sensory afferent that connect all 
resistance reflex Dep MNs would affect all Dep MN responses, whereas presynaptic inhibition of sensory fiber connecting only one Dep MN would only affect this $\mathrm{MN}$ response. Such differential reflex responses have been demonstrated in the thoraco-coxal joint (Skorupski et al. 1992), that could be supported by specific sensory-motor projections such as the one presented here. Functional analysis of divergence of sensory inputs remains a question difficult to unravel. So far, the crayfish thoracic in vitro preparation is one of the rare models which appear to be adapted to solve this problem. Nevertheless, we must keep in mind that results obtained in vitro are oversimplified compared to intact animal in which more neurons would be active, and modulation from central or peripheral origin would be capable of rebuilding neuronal networks (Meyrand et al. 1991).

\section{Role and interpretation of EPSP shape.}

This study has demonstrated that EPSPs from a given CBCO afferent onto different Dep MNs displayed different shapes (time to peak from 0.7 to $1.3 \mathrm{~ms}$, decrease time constant from 2.14 to $12.44 \mathrm{~ms}$, amplitude from 0.07 to $0.5 \mathrm{mV}$ ). Because of the geometry of the MN dendritic trees, EPSP generated on small distant branches would elicit smaller and slower centrally recorded events than the same EPSP generated on proximal branches (Rall 1967; Rall et al. 1967). In order to test this assertion, we have simulated propagation of EPSPs in a compartment model (Fig. 9A). It appeared that temporal characteristics of the propagated EPSP also depends upon the geometrical characteristics of the postsynaptic compartment in which it has been produced: the EPSP duration is directly related to the membrane resistivity, but inversely related to the diameter of the postsynaptic compartment (Fig. 9B). Therefore, in electrophysiological recordings, the variability in the shape of the EPSPs recorded from distinct MNs could be due to MN morphological differences. However, recording different EPSP shapes from the same MN would likely indicate different synaptic locations (see 
differences between proximally and distally generated EPSPs in figure 9B). Then the summation properties of EPSPs in synapses located at various places within the MN dendritic tree, was studied in different conditions of membrane resistivity for both small and large postsynaptic compartment configurations (Fig. 10): here again, the difference between proximal and distal synapses was more pronounced in the large postsynaptic compartment configuration (Fig. 10B cf. A and D cf. C). Except the case of low membrane resistivity $\left(R_{\mathrm{m}}=4 \mathrm{k} \Omega \cdot \mathrm{cm}^{2}\right)$, the simulated frequency stimulation affected the postsynaptic depolarization amplitude in a way very similar to reality (cf. Fig. 8). On the basis of these simulation studies, we propose a functional interpretation of synapse location, that matches to physiological observations: proximal synapses would produce fast, large amplitude EPSPs involved in the MN phasic firing; in opposite, distal synapses would not produce enough depolarization to give rise to an immediate $\mathrm{MN}$ discharge, but rather contribute to a slow graded depolarization that would increase the neuron excitability (see complete fusion of distally generated EPSPs, in figure 10B). However, synapse location is not the only way to account for the variety of EPSP shapes: time to peak, duration and decay time are characteristics that could be specified at the synapse itself. Such parameters would thus depend upon neurotransmitter release probabilities, diffusion coefficient, and uptake/degradation functions that can vary from one synapse to the other (Laurent and Sivaramakrishnan 1992; Redman and Walmsley, 1983).

Functional implications of the resistance reflex design.

In an associated paper, we described that, in the angular sector studied, movements imposed to the $\mathrm{CBCO}$ strand activated the release-sensitive sensory units in a greater number than stretch-sensitive ones. Moreover, although their coding was not so specific, these sensory units were activated in a sequential way (Le Ray et al. 1997). Here, we demonstrated that Dep MNs were also activated sequentially (Fig. 2). In both sensory coding 
and $\mathrm{MN}$ response, the angular sector eliciting the strongest neuronal activation is comprised between the full release (corresponding to the leg contacting the dorsal thoracic carapace) and about 60 degrees in the stretching direction (corresponding to the basipodite around the horizontal position). Although these results were obtained from an isolated nervous system in high $\mathrm{Ca}^{2+}-$ high $\mathrm{Mg}^{2+}$ saline, it is likely that the same sensory-motor pathways are involved in the intact animal; the monosynaptic connections from CBCO afferents onto Dep MNs would then operate to uphold the body in the most commonly observed posture.

In this study, all intracellularly recorded Dep MNs displayed phasic or phasico-tonic responses to $\mathrm{CBCO}$ strand ramp stimulation (Figs. 6 and 7). The absence of purely tonic $\mathrm{MN}$ response is the consequence of the absence of purely position-coding $\mathrm{CBCO}$ afferent (Le Ray et al. 1997). Because they receive several release-sensitive CBCO afferents (Fig. 3), all coding for release movements, all resistance Dep MNs presented larger reflex responses during movement than during maintained position (Figs. 4, 6 and 7). This mainly dynamic feedback would therefore operate as a zero-velocity servo control: it is not the position but the movement that is detected in order to maintain a constant position. We could have think that the resistance reflex, mainly involved in posture, would have been based on the position coding. In contrary, the crayfish walking network chose a more "simple" system which does not need to compare permanently to precise position-coding references. Moreover, a system only based upon position-coding would require an incompressible integration time to take into account, and counteract, changes in position. The strategy adopted in the crayfish sensorymotor system allows faster dynamically-adapted responses. Therefore, in resting animal, the resistance reflex pathway would only be activated if a perturbation occurs. How this neuronal pathway, here involved in purely negative feedback, would work during more complex motor behaviors in intact animal remains to be determined. 


\section{REFERENCES}

Barnes, W. J. P., And Gladden, M. H., eds. Feedback and Motor Control in Invertebrates and Vertebrates. London, Croom Helm, 1985.

BÄSSLER, U. The femur tibia control system of stick insects. A model system for the study of the neural basis of joint control. Brain Res. Rev. 18: 207-226, 1993.

Berry, M. S., AND Pentreath, V. W. Criteria for distinguishing between monosynaptic and polysynaptic transmission. Brain Res. 105: 1-20, 1976.

Blight, A. R., AND LlinAS, R. The non-impulsive stretch-receptor complex of the crab - a study of depolarization-release coupling at a tonic sensorimotor synapse. Philos. Trans. R. soc. London (Biol) 290: 219-276, 1980.

Burke, R. E., AND Rudomin, P. Spinal neurons and synapses. In: Handbook of Physiology. The nervous system. Cellular biology of neurons. Section 1, vol. 1, part 2, chap. 24, edited by J. M. Brookhart and V. B. Mountcastle, Bethesda, Amer. Physiol. Soc., 1977, p. 877-944.

BuRROws, M. Parallel processing of proprioceptive signals by spiking local interneurons and motor neurones in the locust. J. Neurosci. 7: 1064-1080, 1987.

Burrows, M., AND PflÜGERS, H. J. Positive feedback loops from proprioceptors involved in leg movements of the locust. J. Comp. Physiol. 163: 425-440, 1988. 
BÜSCHGES, A., AND WOLF, H. Nonspiking local interneurons in insect leg motor control. I. Common layout and species-specific response properties of femur-tibia joint control pathways in stick insect and locust. J. Neurophysiol. 73: 1843-1860, 1995.

Bush, B. M. H. Proprioception by the coxo-basal chordotonal organs, CB, in legs of the crab, Carcinus maenas. J. Exp. Biol. 42: 285-297, 1965a.

Bush, B. M. H. Leg reflexes from chordotonal organs in the crab, Carcinus maenas. Comp. Biochem. Physiol. 15: 567-587, 1965 b.

Cattaert, D., El Manira, A., And Clarac, F. Direct evidence for presynaptic inhibitory mechanisms in crayfish sensory afferents. J. Neurophysiol. 67: 610-624, 1992.

Clarac, F., Chrachri, A., AND CATTAert, D. Interneuronal control of a walking leg reflex in an in vitro crayfish preparation. In : Locomotor neural mechanism in arthropods and vertebrates edited by D. M. Armstrong and B. M. H. Bush. Manchester and New York, Manchester University Press, 1991, p. 33-50.

ECCLES, J. C. The physiology of nerve cells. Baltimore: John Hopkins Press, 1957.

ECCLES, J. C. Functional organization of the spinal cord. Anesthesiology 28: 31-44, 1967.

ECCles, J. C., EcCles, R. M., AND LundberG, A. The convergence of monosynaptic excitatory afferents on to many different species of alpha motoneurones. J. Physiol. 137: 22$50,1957$. 
Eccles, J. C., Schmidt, R. F., AND Willis, W. D. Presynaptic inhibition of the spinal monosynaptic reflex pathway. J. Physiol. 161: 282-297, 1962.

Eckeberg, Ö., Wallen, P., Lansner, A., Traven, H., Brodin, L., And Grillner, S. A computer based model for realistic simulations of neural networks I. The single neuron and synaptic interaction. Biol. Cybernetics 65: 1-90, 1991.

El Manira, A., Cattaert, D., ANd Clarac, F. Monosynaptic connections mediate resistance reflex in crayfish (Procambarus clarkii) walking legs. J. Comp. Physiol. 168: 337-349, 1991.

GRANIT, R. Receptors and sensory perception. New Haven, Connecticut, Yale University Press, 1955.

Harris-Warrick, M., Marder, E., Selverston, A. I., And Moulins, M. Dynamic biological networks: The stomatogastric nervous system. MIT press, Cambridge, Massachusetts, 1992.

LAurent, G., AND Sivaramakrishnan, A. Single local interneurons in the locust make central synapses with different properties of transmitter release on distinct postsynaptic neurons. J. Neurosci. 12: 2370-2380, 1992.

Le RAY, D., AND CATTAert, D. Neural Mechanisms of reflex reversal in coxo-basipodite depressor motor neurons of the crayfish. J. Neurophysiol., in press, 1997. 
Le Ray, D., Clarac, F., AND CATtaert, D. Functional analysis of the sensory motor pathway of resistance reflex in crayfish. II- Multi-sensory coding and motor neuron monosynaptic responses. J. Neurophysiol., in press, 1997.

LOCKERY, S. R., AND KRISTAN, W. B. Distributed processing of sensory information in the leech. I. Input-output relations of the local bending reflex. J. Neurosci. 10: 1811-1815, 1990.

MATHESON, T. Responses and locations of neurones in the locust metathoracic femoral organ. J. Comp. Physiol. A 166: 915-927, 1990.

Matthews, P. B. C. Mammalian muscle receptors and their central actions. Edward Arnold, London, 1972.

Meyrand, P., Simmers, J., AND Moulins, M. Construction of a pattern-generating circuit with neurons of different networks. Nature (Lond) 351: 60-63, 1991.

Nelson, S. G., AND Mendell, L. M. Projection of single knee flexor Ia fibers to homonymous and heteronymous motorneurons. J. Neurophysiol. 41: 778-787, 1978.

NeWland, P. L., AND Nagayama, T. Parallel processing of proprioceptive information in the terminal abdominal ganglion of the crayfish. J. Comp. Physiol. A 172: 389-400, 1993.

RALL, W. Distinguishing theoretical synaptic potentials computed for different soma-dendritic distributions of synaptic input. J. Neurophysiol. 30: 1138-1168, 1967. 
Rall, W., Burke, R. E., Smith, T. G., Nelson, P. G., Frank, K. Dendritic location of synapses and possible mechanisms for the monosynaptic EPSP in motoneurons. J. Neurophysiol. 30: 1169-1193, 1967.

REDMAN, S. J. Junctional mechanisms at group Ia synapses. Prog. Neurobiol. 12: 33-83, 1979.

Redman, S. J., AND Walmsley, B. Amplitude fluctuations in synaptic potentials evoked in cat spinal motoneurones at identified group Ia synapses. J. Physiol. 343: 135-145, 1983.

Sauer, A. E., Driesang, R. B., BÜschges, A., AND BäSSLER, U. Distributed processing on the basis of parallel and antagonistic pathways simulation of the femur-tibia control system in the stick insect. J. Computational Neurosci. 3: 179-198, 1996.

ScotT, J. G., AND Mendell, L. M. Individual EPSPs produced by single triceps surae Ia afferent fibers in homonymous and heteronymous motoneurons. J. Neurophysiol. 39: 679-692, 1976.

SHERRINGTON, C. S. Flexion-reflex of the limb, crossed extension reflex stepping and standing. J. Physiol. (Lond) 40: 28-121, 1910.

SkORUPSKI, P., RAWAT, B. M., AND Bush, B. M. H. Heterogeneity and central modulation of feedback reflexes in crayfish motor pool. J. Neurophysiol. 67: 648-663, 1992. 


\section{LEGENDS}

Fig. 1: Diagram of the experimental procedure - Methodology.

A: Drawing of the neurons involved in the resistance reflex pathway. Single or paired intracellular recordings from sensory terminal (CBT) and depressor motor neuron (Dep MN) are performed to analyze their connections. B: In high calcium - high magnesium saline, monosynaptic connections are preserved. C: Procedure used to analyze the different sensory units recorded from the $\mathrm{CBCO}$ nerve, and their connections with Dep MNs. In this illustration, five distinct sensory units were identified by their shape and amplitude, and produced EPSPs in some of the three postsynaptic Dep MNs represented (A MN: assistance Dep MN; R MNs: two distinct resistance Dep MNs). The occurrence of each sensory unit (unit 1 in the example) within the stimulation cycle was represented using circular statistics by an unit vector $\left(\vec{v}_{i}\right)$. The vectorial sum of these unit vectors was calculated over several cycles and divided by the number (n) of occurrences: the resulting vector $(\vec{R})$ indicated the mean response of the sensory unit (see text for details). This procedure is repeated for each sensory afferent presynaptic to the considered MN (R MN1 in the example).

Table 1: Connections between sensory fibers and Dep MNs.

On the basis of the results obtained with circular statistics from several experiments, we classified the CBCO units that connected the Dep MNs according to their reflex response ( $\varnothing$ : non-responding; A: assistance; R: resistance, in the first column). The number of afferents and their specificity to movement (release, non-specific and stretch) are indicated for each Dep MN.

Fig. 2: Temporal distribution of sensory-motor connections. 
This example was obtained in one experiment in which all Dep MNs were successively impaled. Only the nine reflex responding Dep MNs are represented. The circular representation displays the resulting vectors of each sensory unit that connect a single $\mathrm{MN}$ (thin lines within the circle). A general resulting vector was calculated for each Dep MN (thick line out of the circle). Temporal distribution of CBCO afferents was variable from one Dep MN to the others. The sine wave diagrams represent for each Dep MN the range of movement in which it receives most of the EPSPs.

Fig. 3: CBCO-related EPSPs in the resistance Dep MNs.

In an experiment in which all resistance Dep MNs were successively impaled, we identified from the CBCO neurogram 14 sensory units that produced an EPSP in the postsynaptic Dep MNs. Each trace represent an average over more than 20 occurrences (except traces marked with ${ }^{1}$ which were averaged over six occurrences). Single CBCO unit-related EPSPs present different shape and amplitude in the different postsynaptic Dep MNs. Vertical bars for all recordings were $0.4 \mathrm{mV}$, except traces marked with a ${ }^{*}(0.8 \mathrm{mV})$.

Fig. 4: Intracellular demonstration of the sensory input divergence.

Successive paired recordings were performed from one release-sensitive phasico-tonic CBT (top) and from all 12 Dep MNs successively impaled (and identified by their extracellular profile, central column). Five distinct Dep MNs, phasic or phasico-tonic (left column), were found to be monosynaptically connected by the same CBT. Averaged EPSPs (right column) present different shapes and amplitudes.

Fig. 5: Control of EPSP frequency - Methodology. 
A: During paired intracellular recording, high frequency intracellular stimulation of one CBT produces compound depolarization in the postsynaptic Dep MN. B: The monosynaptic transmission of information follows one-to-one the stimulation frequency: stimulation of each CBT produces an identifiable EPSP in the Dep MN. C: CBCO-related EPSPs are very variable in amplitude, time to peak and decay time. As an example, the relative exponential decay of repolarizing phase from six representative EPSPs are plotted as a function of time. The time constant $(\tau)$ of the repolarizing phase of each CBCO-related EPSP was calculated by one-phase exponential decay fitting: although distributed between two extreme values $(2.5 \mathrm{~ms}$ and $12.5 \mathrm{~ms}$ ), the mean EPSP $\tau$ value was $6.5 \mathrm{~ms}$.

Fig. 6: Phasic Dep MN response to high frequency stimulations of a single CBT.

A: The phasic Dep MN response to CBCO strand imposed ramp movement is characterized by phasic bursts of EPSPs occurring exclusively during releasing movements. B: In the absence of movement, phasically-applied high frequency intracellular stimulation of a single presynaptic CBT is able to reproduce a phasic-like response in the Dep MN (1). Phasicotonic-like response could be elicited in the Dep MN by adjusting tonic stimulations between the $40 \mathrm{~Hz}$-stimulations (2).

Fig. 7: Phasico-tonic Dep MN response to high frequency stimulations of a single CBT.

A: The phasico-tonic Dep MN response to CBCO strand imposed ramp movement, is characterized by a sustained membrane depolarization during maintained released positions, and phasic bursts of EPSPs during releasing movements. B: In the absence of movement, the frequency of intracellular stimulation of a single presynaptic CBT was adjusted in order to reproduce a phasico-tonic-like response in the Dep MN (1). In the same paired recording, the phasico-tonic response could be change into a perfect phasic-like response in the Dep MN (2). 
Fig. 8: Time course of compound EPSPs elicited by train-stimulation of a single CBT.

A: Phasic response Dep MN. The plot of the depolarization amplitude as a function of time (top) shows the marked frequency-dependence (compare 50 and $200 \mathrm{~Hz}$ ) of the compound EPSP. The same intracellular stimulation frequency $(200 \mathrm{~Hz})$ applied to distinct presynaptic CBTs, produces different compound EPSP shapes in the Dep MN (bottom). B: Phasico-tonic response Dep MN. Frequency-dependence of the compound EPSP (top) and compound EPSP shapes produced by distinct presynaptic CBT stimulation (bottom), are comparable to those observed in a phasic Dep MN.

Fig. 9: Simulation of single EPSPs - Consequence of the postsynaptic compartment geometry. A: Diagrams of the compartment model configurations used in this study. The diameter of the distal postsynaptic compartment varied from $20 \mu \mathrm{m}$ (left) to $1 \mu \mathrm{m}$ (right). Synapses were multiple and distally located (d), or unique and centrally located (p). The resulting EPSP was recorded in the same compartment of the main neurite. In both configurations, various membrane resistivities were tested $\left(4,8,12\right.$, and $\left.20 \mathrm{k} \Omega . \mathrm{cm}^{2}\right)$. B: The effect of the membrane resistivity (Rm) upon the peak amplitude, time to peak and time constant (Tau) of the simulated EPSP is presented on three graphs (see text for details). Squares: $20 \mu \mathrm{m}$ in diameter configuration; circles: $1 \mu \mathrm{m}$ in diameter configuration; filled symbols: distal synapses; unfilled symbols: proximal synapses.

Fig. 10: Simulation of trains of EPSPs.

The two configurations (Diam $=1 \mu \mathrm{m}$ or $20 \mu \mathrm{m}$, cf. Fig. 9A) were tested with various membrane resistivities ( $\mathrm{Rm})$, upon trains of distally (left) or proximally (right) generated EPSPs. Three frequencies were used $(140,80$, and $50 \mathrm{~Hz})$ in each condition. Raw data are 
presented in each case for 50 and $140 \mathrm{~Hz}$-stimulations. Individual EPSPs have been normalized (100\%) in order to allow direct comparison of the time course of all EPSP trains. Only the extreme conditions are presented: A: Small postsynaptic compartments and Rm of $20 \mathrm{k} \Omega . \mathrm{cm}^{2}$; B: Large postsynaptic compartments and $\mathrm{Rm}$ of $20 \mathrm{k} \Omega . \mathrm{cm}^{2}$; C: Small postsynaptic compartments and $\mathrm{Rm}$ of $4 \mathrm{k} \Omega . \mathrm{cm}^{2}$; D: Large postsynaptic compartments and $\mathrm{Rm}$ of $4 \mathrm{k} \Omega \cdot \mathrm{cm}^{2}$. Physiological compound EPSPs were found between these extreme simulations (see text for details). 
A

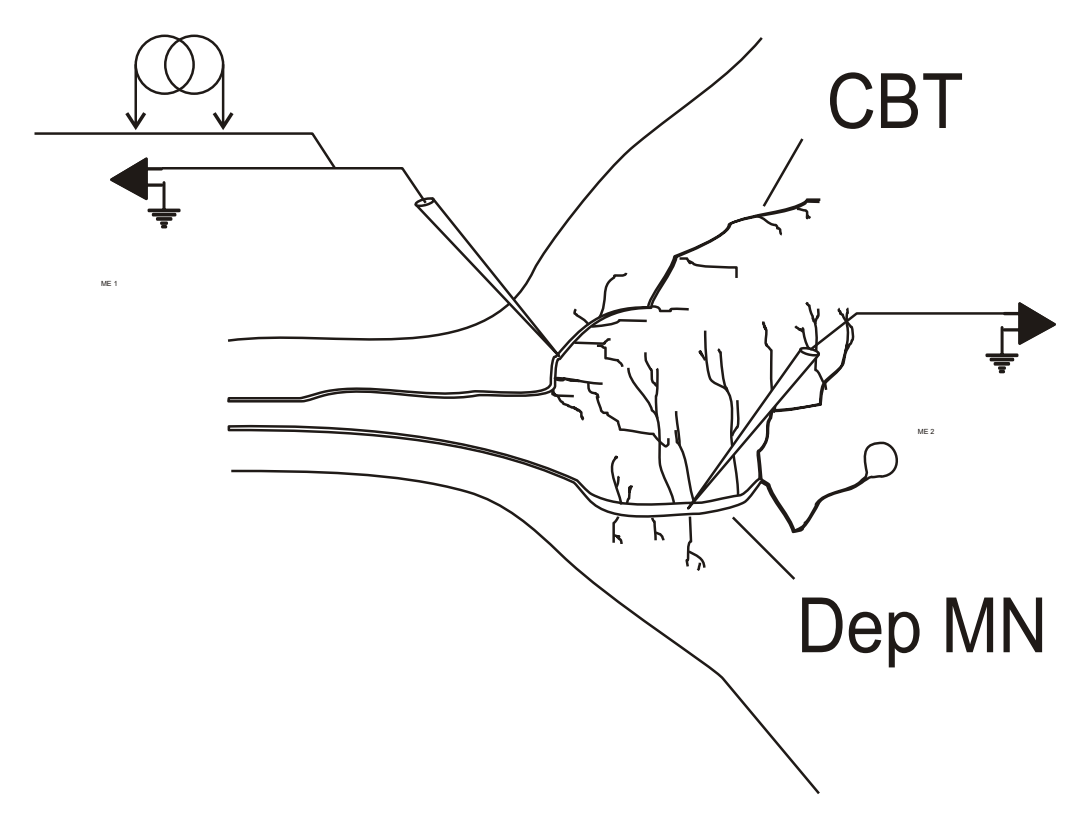

B
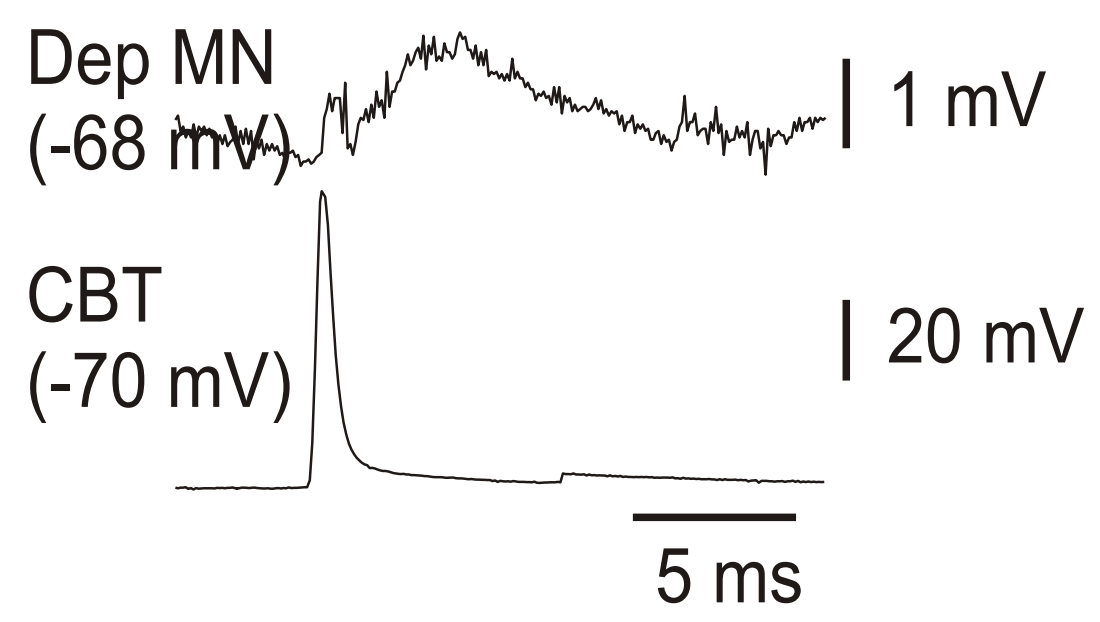

$C_{1}$

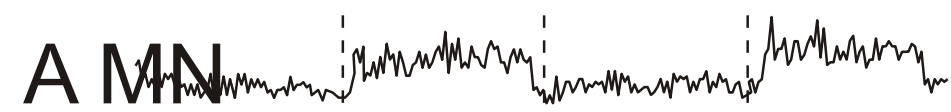

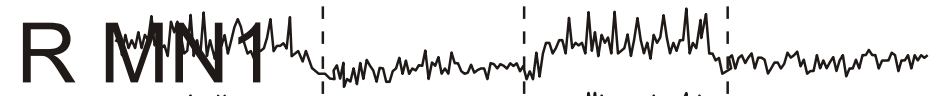

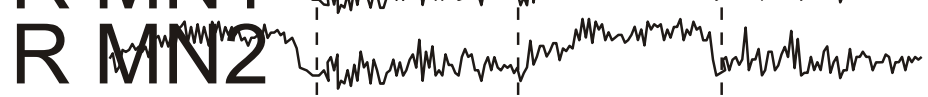

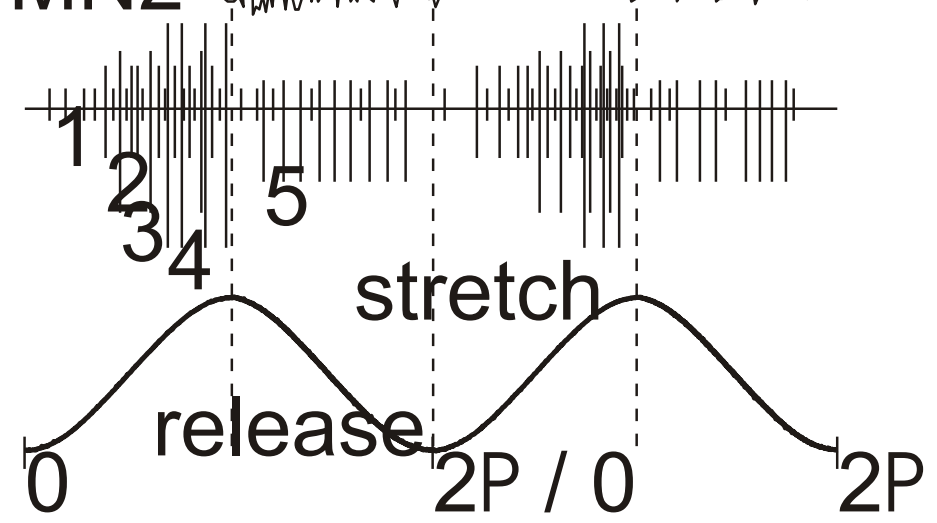

2

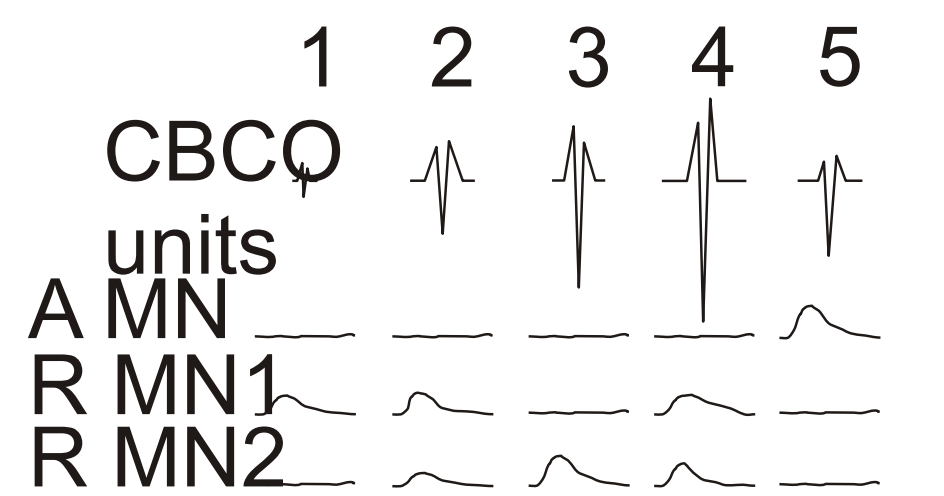

3

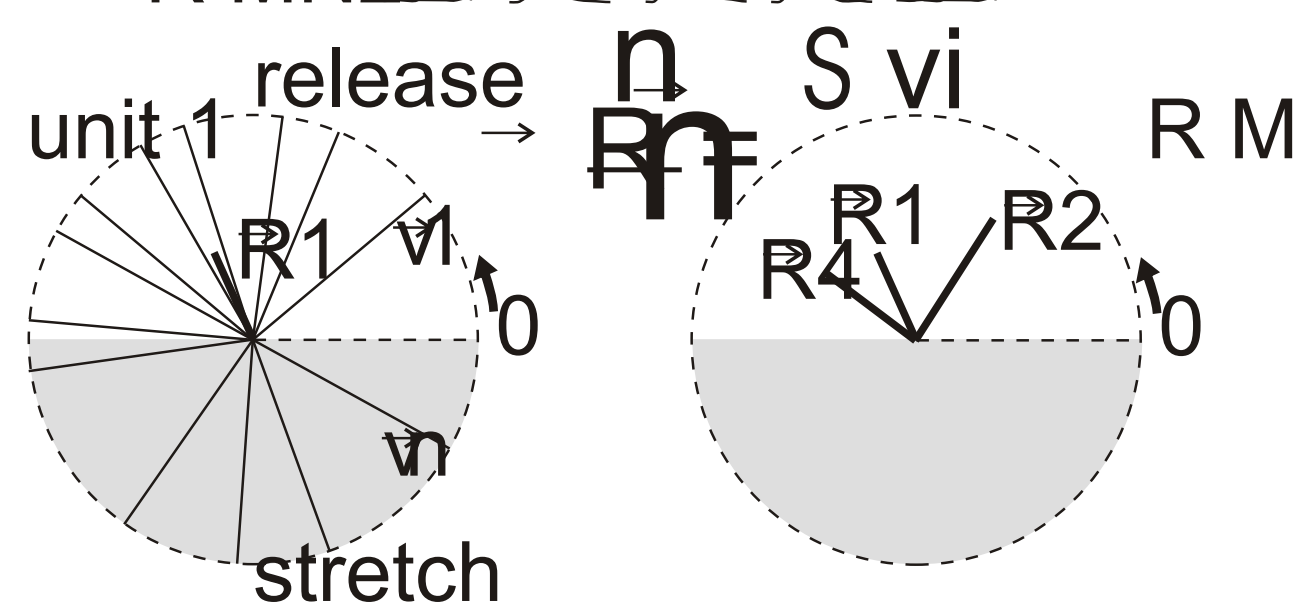




\begin{tabular}{|c|c|c|c|c|c|}
\cline { 4 - 6 } \multicolumn{1}{c|}{} & type of & number of & \multicolumn{3}{c|}{ CBCO unit type } \\
\cline { 4 - 6 } \multicolumn{1}{c|}{ response } & CBCO units & Release & Non specific & Stretch \\
\hline MN 1 & $\varnothing$ & 0 & 0 & 0 & 0 \\
\hline MN 2 & $\varnothing$ & 0 & 0 & 0 & 0 \\
\hline \hline MN 3 & $\mathrm{A}$ & 5 & 0 & 0 & 5 \\
\hline MN 4 & $\mathrm{A}$ & 4 & 0 & 0 & 4 \\
\hline MN 5 & $\mathrm{A}$ & 4 & 0 & 0 & 4 \\
\hline \hline MN 6 & $\mathrm{R}$ & 5 & 5 & 0 & 0 \\
\hline MN 7 & $\mathrm{R}$ & 7 & 6 & 1 & 0 \\
\hline MN 8 & $\mathrm{R}$ & 7 & 4 & 3 & 0 \\
\hline MN 9 & $\mathrm{R}$ & 5 & 5 & 0 & 0 \\
\hline MN 10 & $\mathrm{R}$ & 4 & 4 & 0 & 0 \\
\hline MN 11 & $\mathrm{R}$ & 5 & 5 & 0 & 0 \\
\hline MN 12 & $\mathrm{R}$ & 4 & 4 & 0 & 0 \\
\hline MN 13 & $\mathrm{R}$ & 7 & 6 & 1 & 0 \\
\hline MN 14 & $\mathrm{R}$ & 6 & 6 & 0 & 0 \\
\hline MN 15 & $\mathrm{R}$ & 6 & 4 & 2 & 0 \\
\hline MN 16 & $\mathrm{R}$ & 5 & 3 & 2 & 0 \\
\hline MN 17 & $\mathrm{R}$ & 6 & 5 & 0 & 1 \\
\hline MN 18 & $\mathrm{R}$ & 6 & 5 & 1 & 0 \\
\hline MN 19 & $\mathrm{R}$ & 3 & 3 & 0 & 0 \\
\hline MN 20 & $\mathrm{R}$ & 7 & 5 & 2 & 0 \\
\hline MN 21 & $\mathrm{R}$ & 4 & 3 & 1 & 0 \\
\hline MN 22 & $\mathrm{R}$ & 7 & 5 & 1 & 1 \\
\hline MN 23 & $\mathrm{R}$ & 6 & 5 & 0 & 1 \\
\hline MN 24 & $\mathrm{R}$ & 4 & 4 & 0 & 0 \\
\hline MN 25 & $\mathrm{R}$ & 6 & 4 & 2 & 0 \\
\hline MN 26 & $\mathrm{R}$ & 6 & 5 & 1 & 0 \\
\hline MN 27 & $\mathrm{R}$ & 5 & 2 & 3 & 0 \\
\hline MN 28 & $\mathrm{R}$ & 4 & 3 & 1 & 0 \\
\hline MN 29 & $\mathrm{R}$ & 3 & 2 & 1 & 0 \\
\hline MN 30 & $\mathrm{R}$ & 7 & 4 & 2 & 1 \\
\hline MN 31 & $\mathrm{R}$ & 7 & 7 & 0 & 0 \\
\hline & & & & & \\
\hline
\end{tabular}




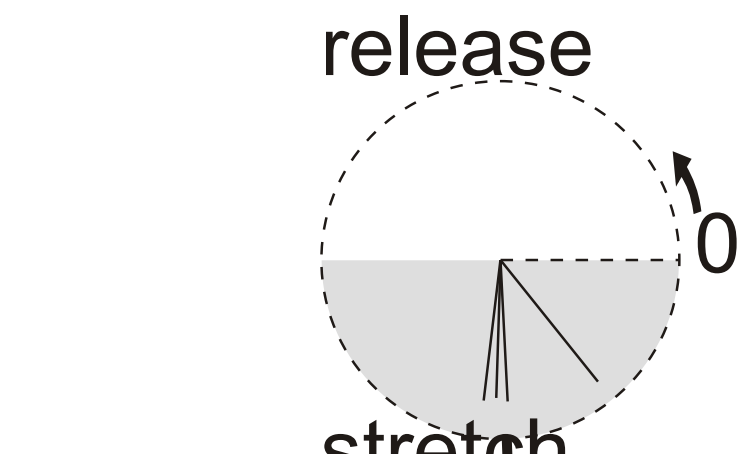

release
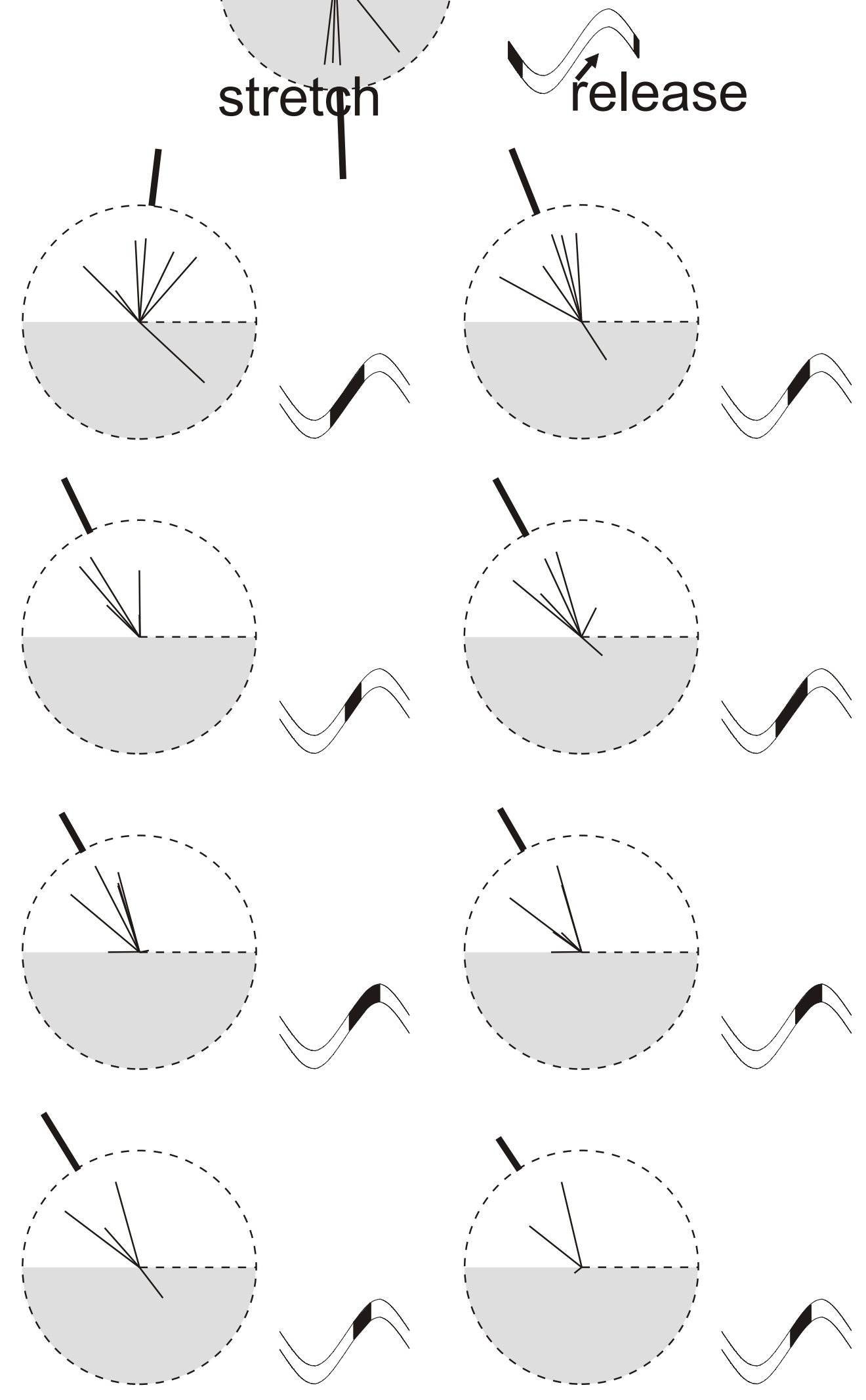


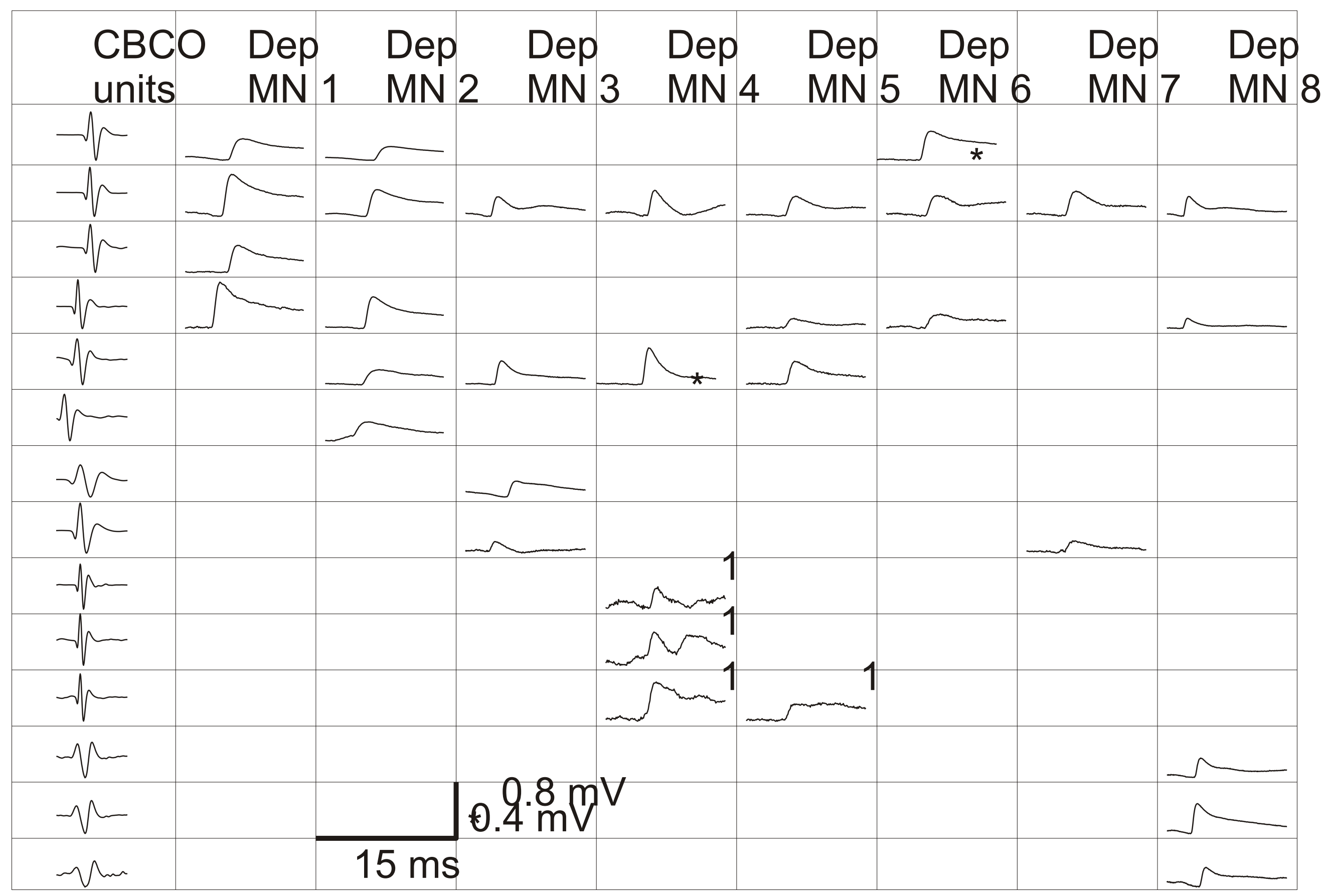



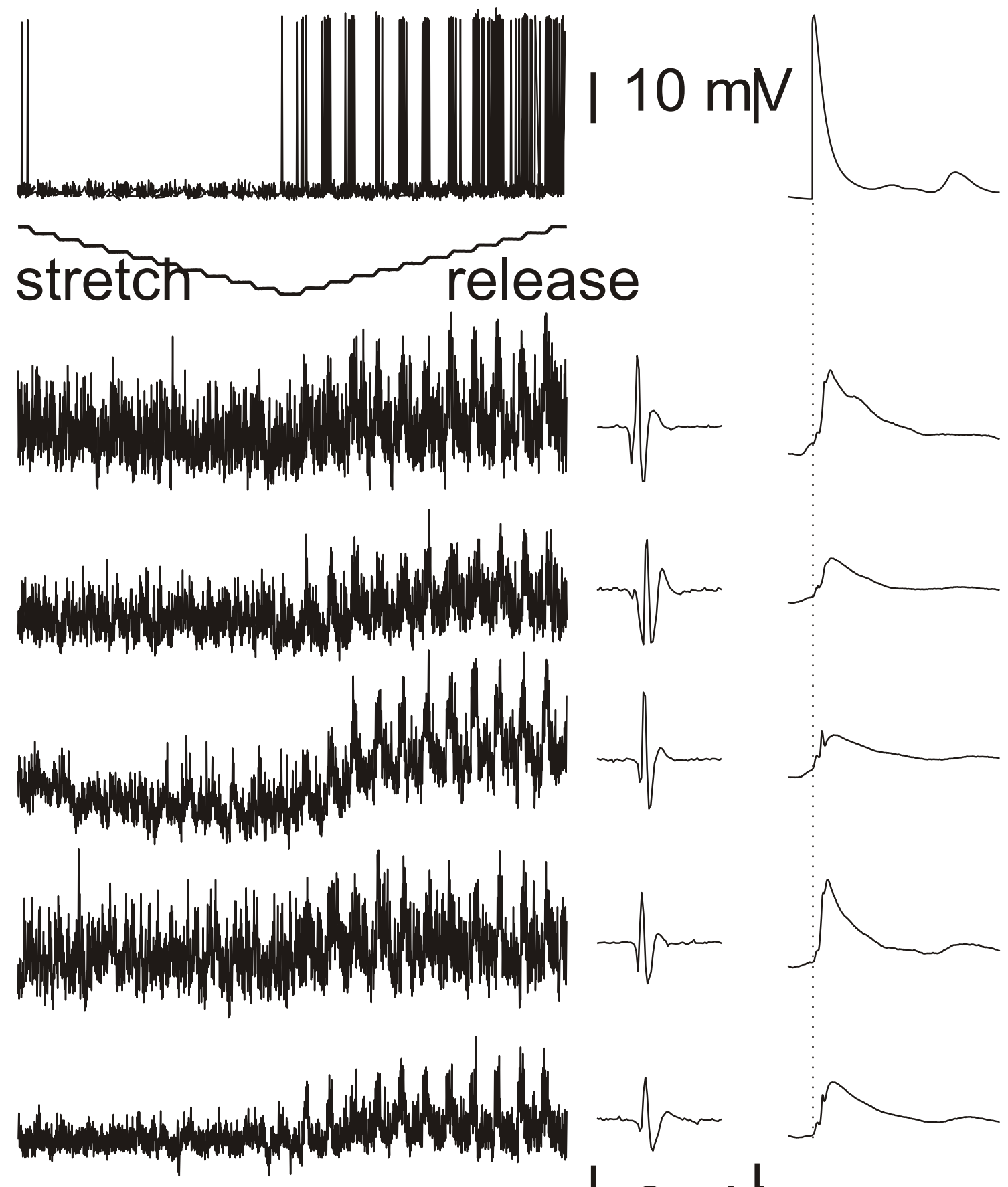

$$
{ }_{5 \mathrm{~s}} 2 \mathrm{my} / 10 \mathrm{~ms}
$$



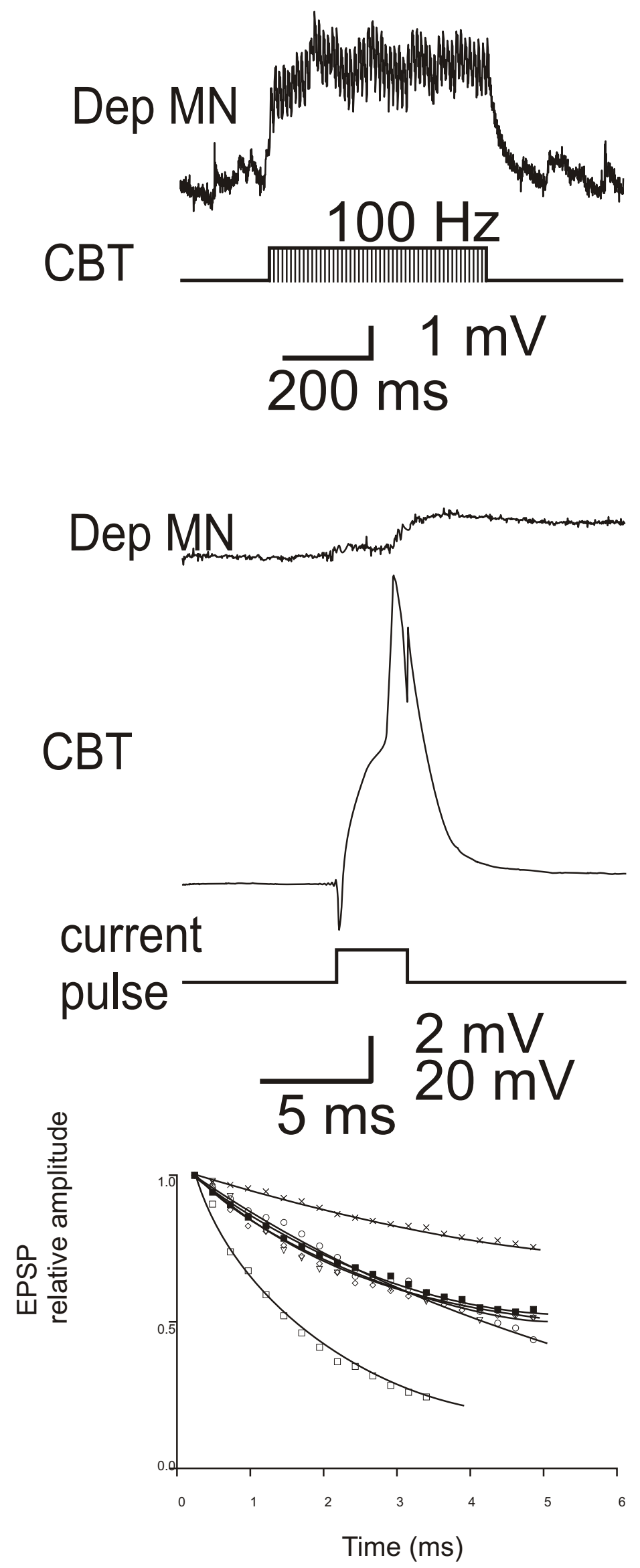
A

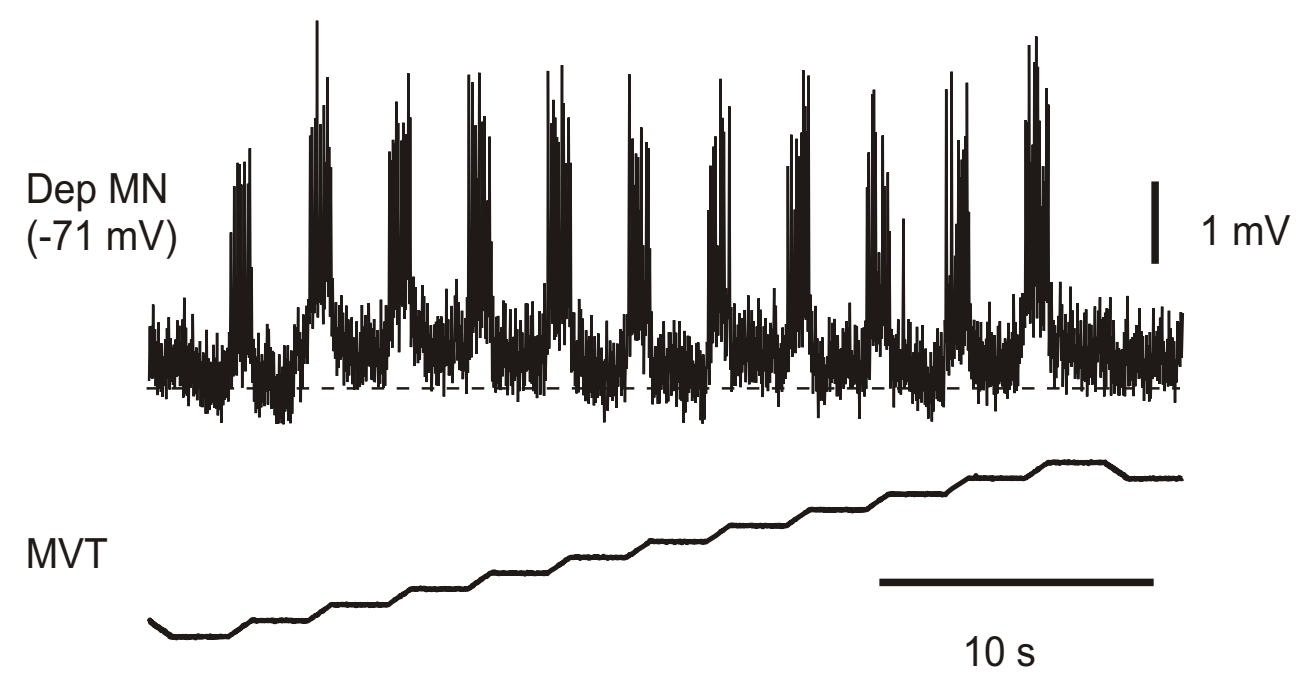

B
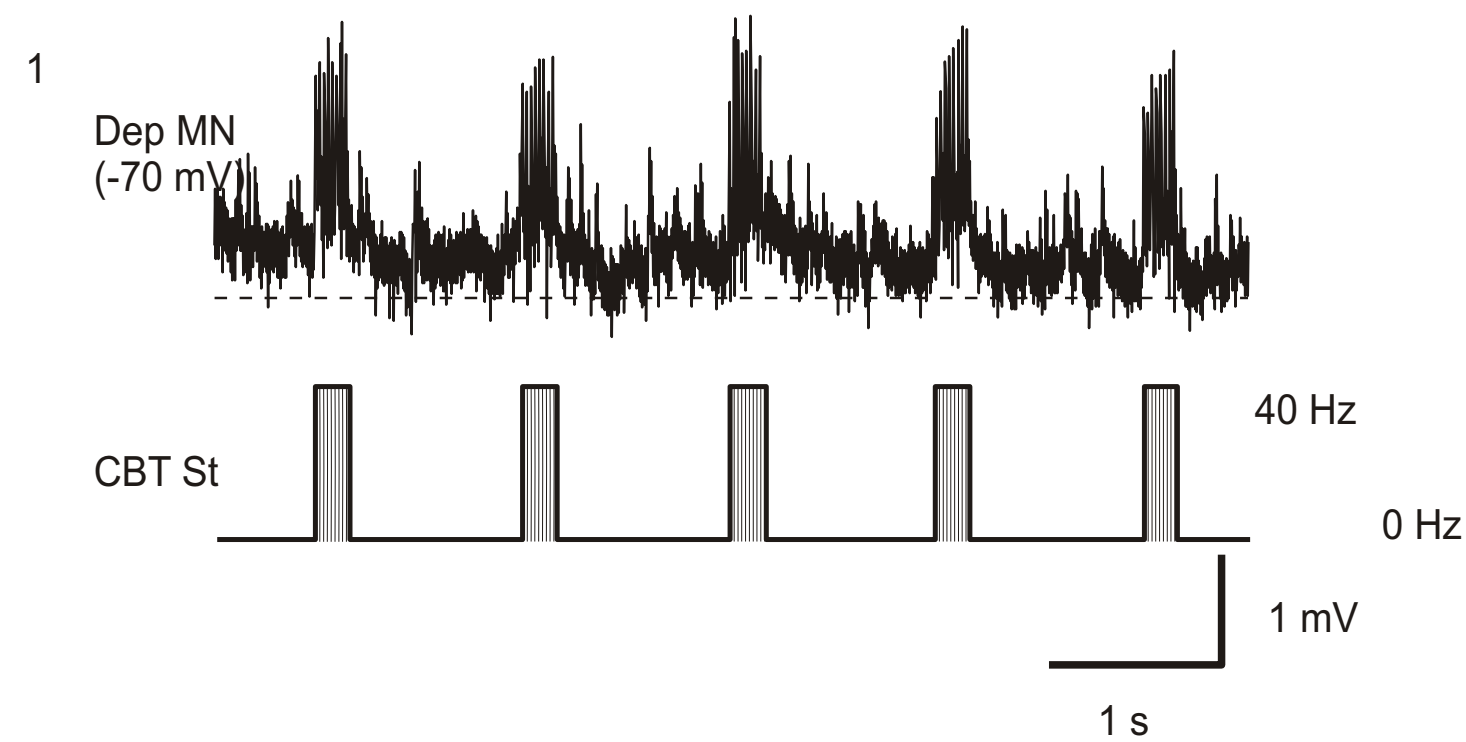

2

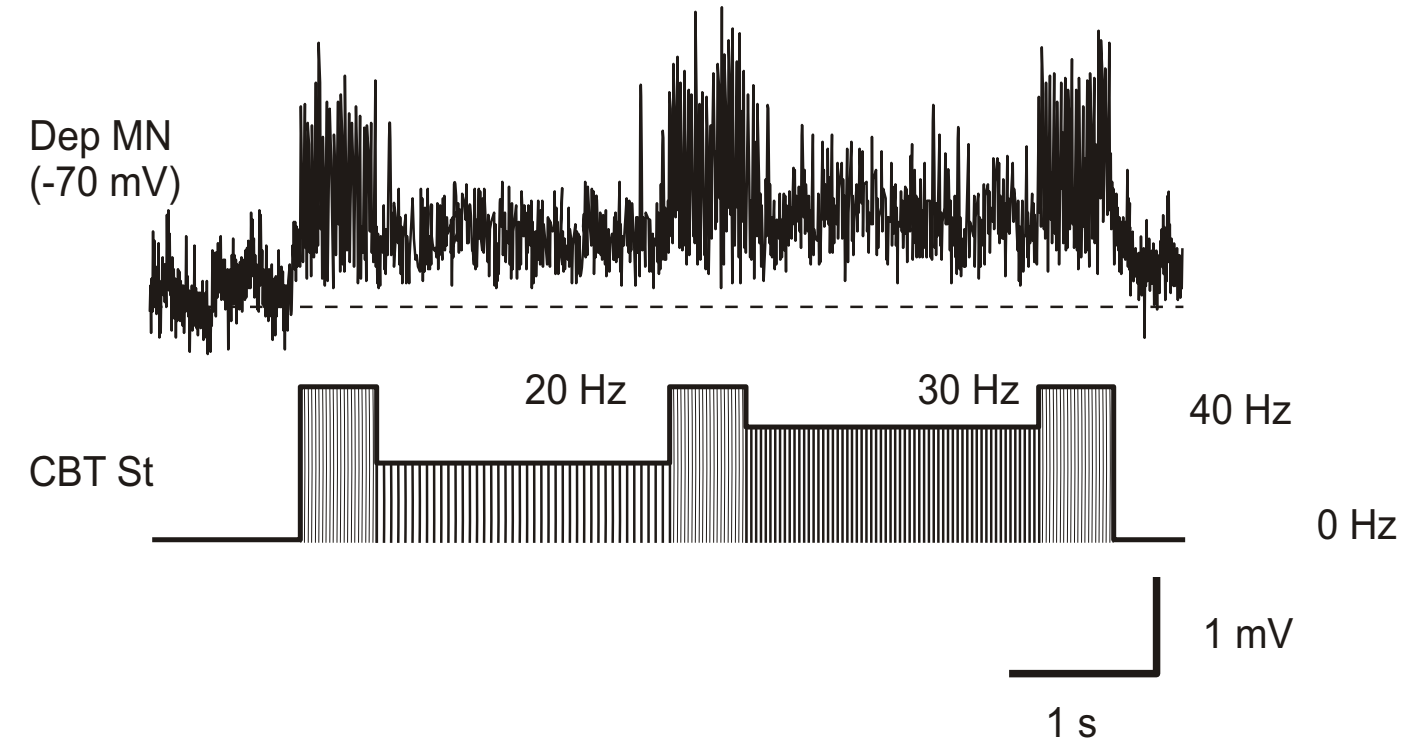


A

B

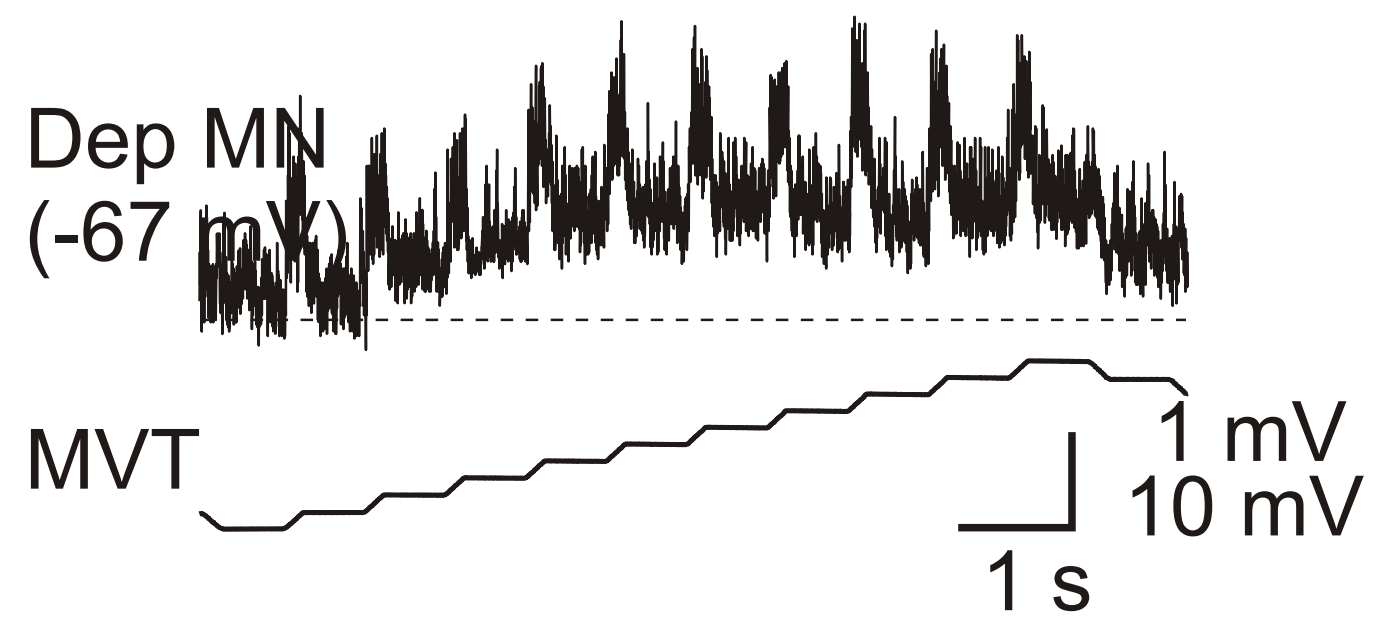

1

Dep MN

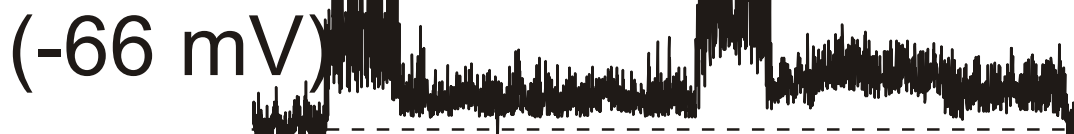

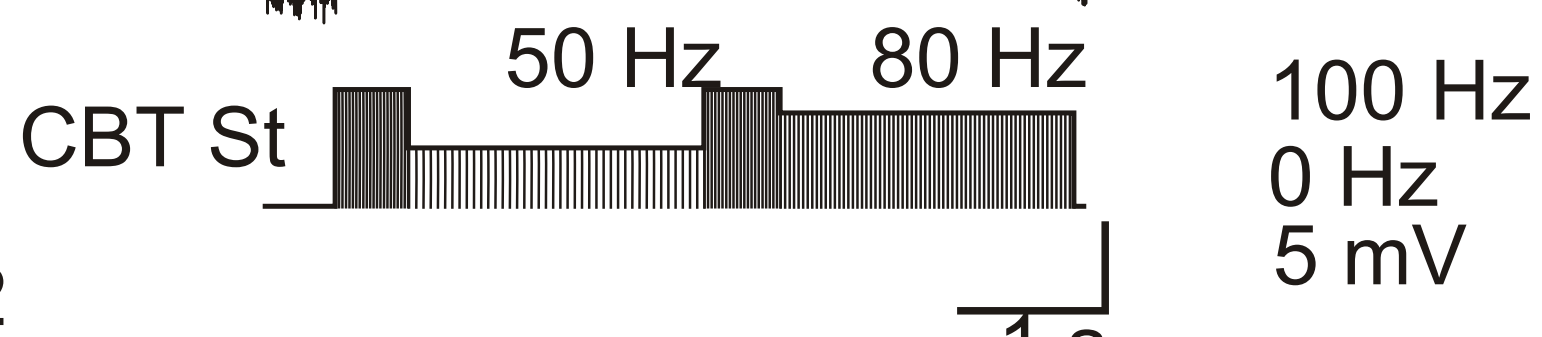

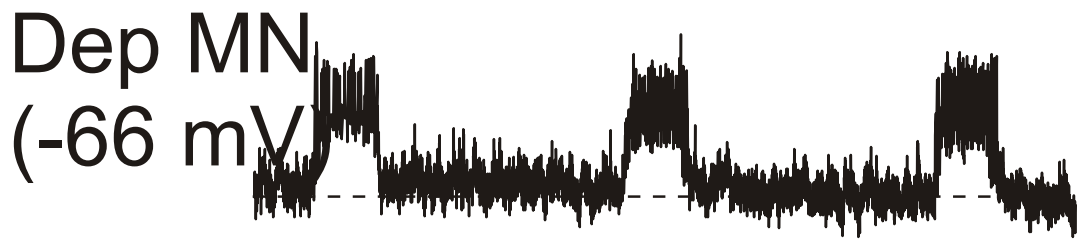

CBT St

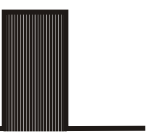

$100 \mathrm{~Hz}$

$0 \mathrm{~Hz}$

$1 \mathrm{~s}$

$5 \mathrm{mV}$ 
A Phasic response Dep MN
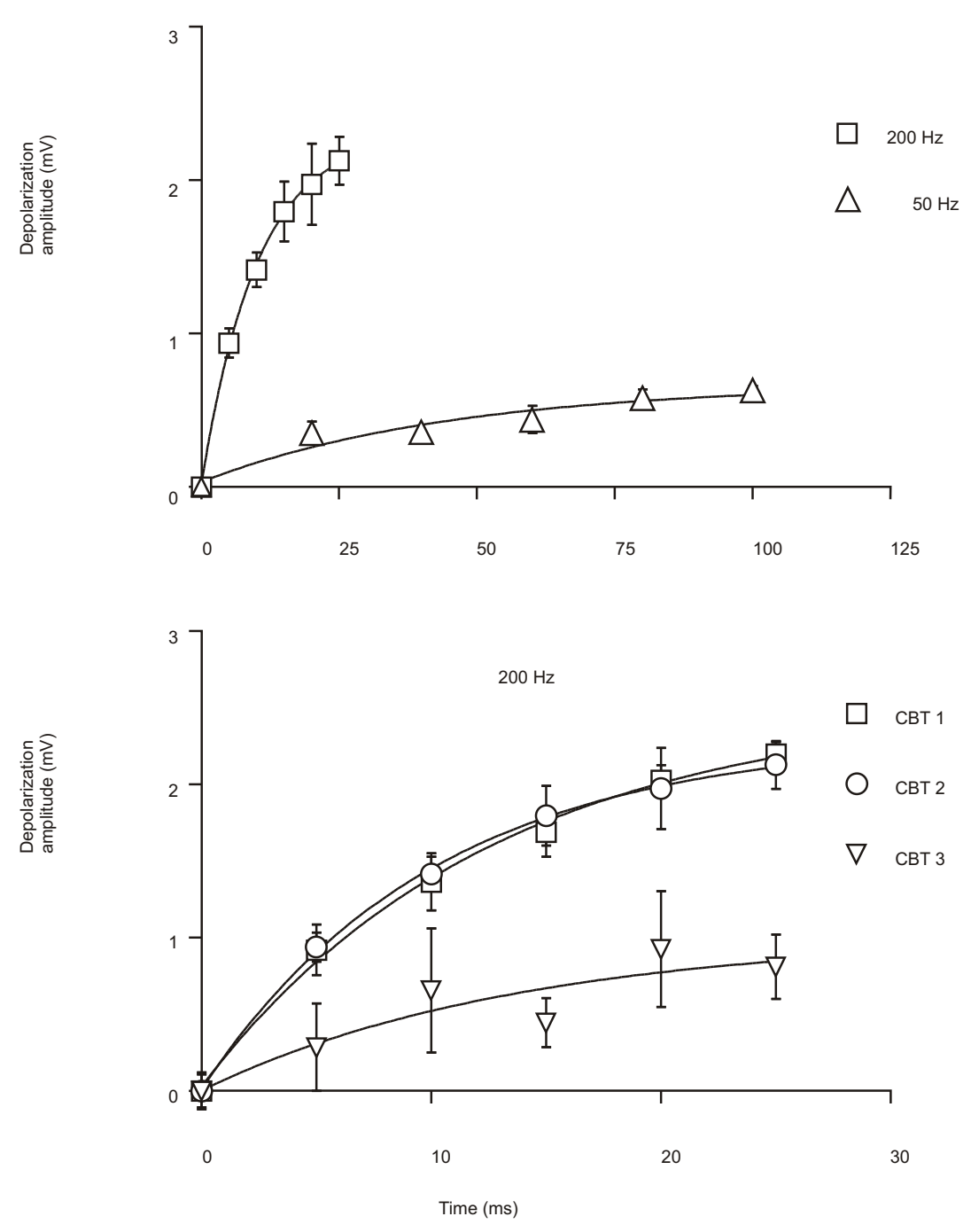

B Phaso-tonic response Dep MN
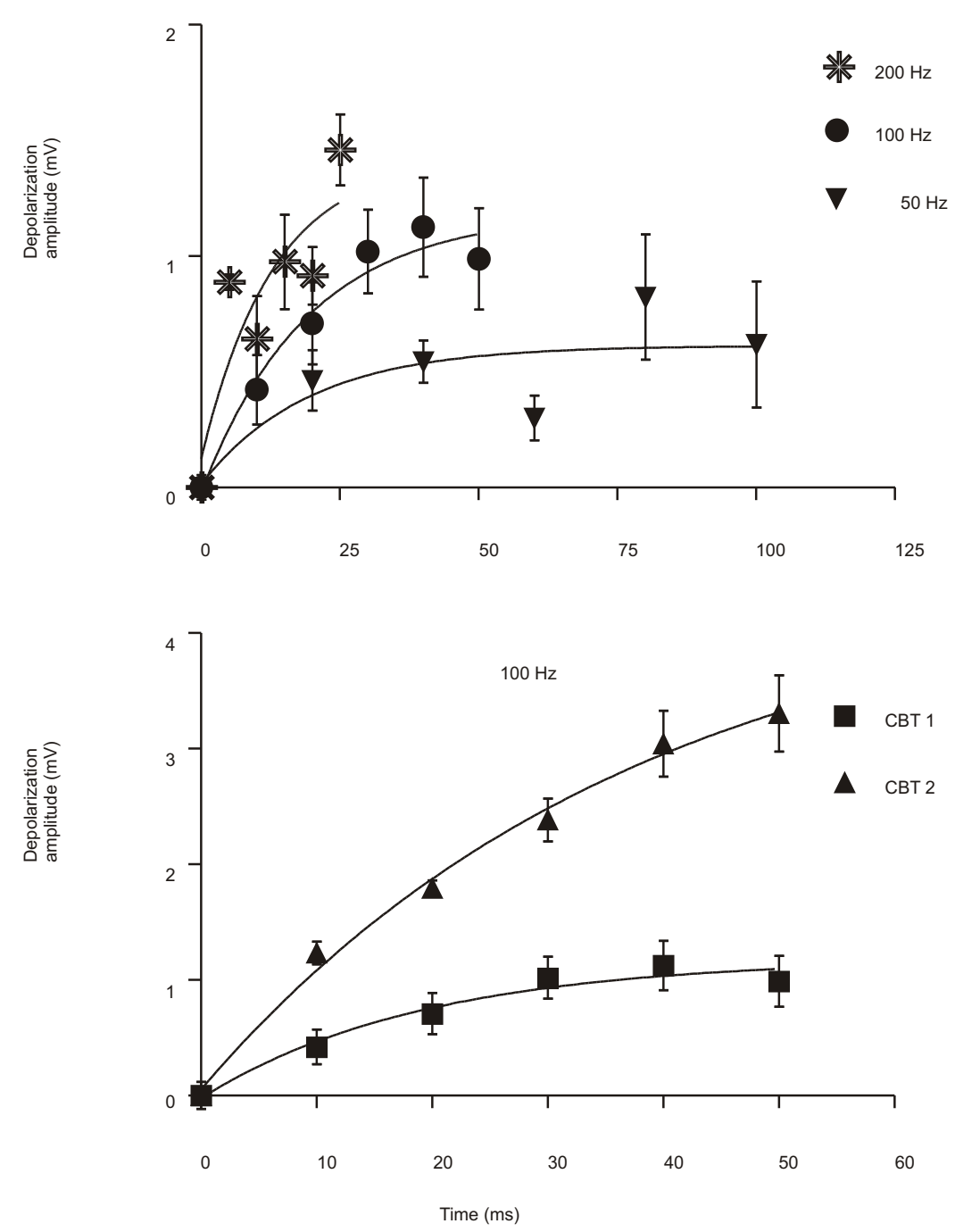
A
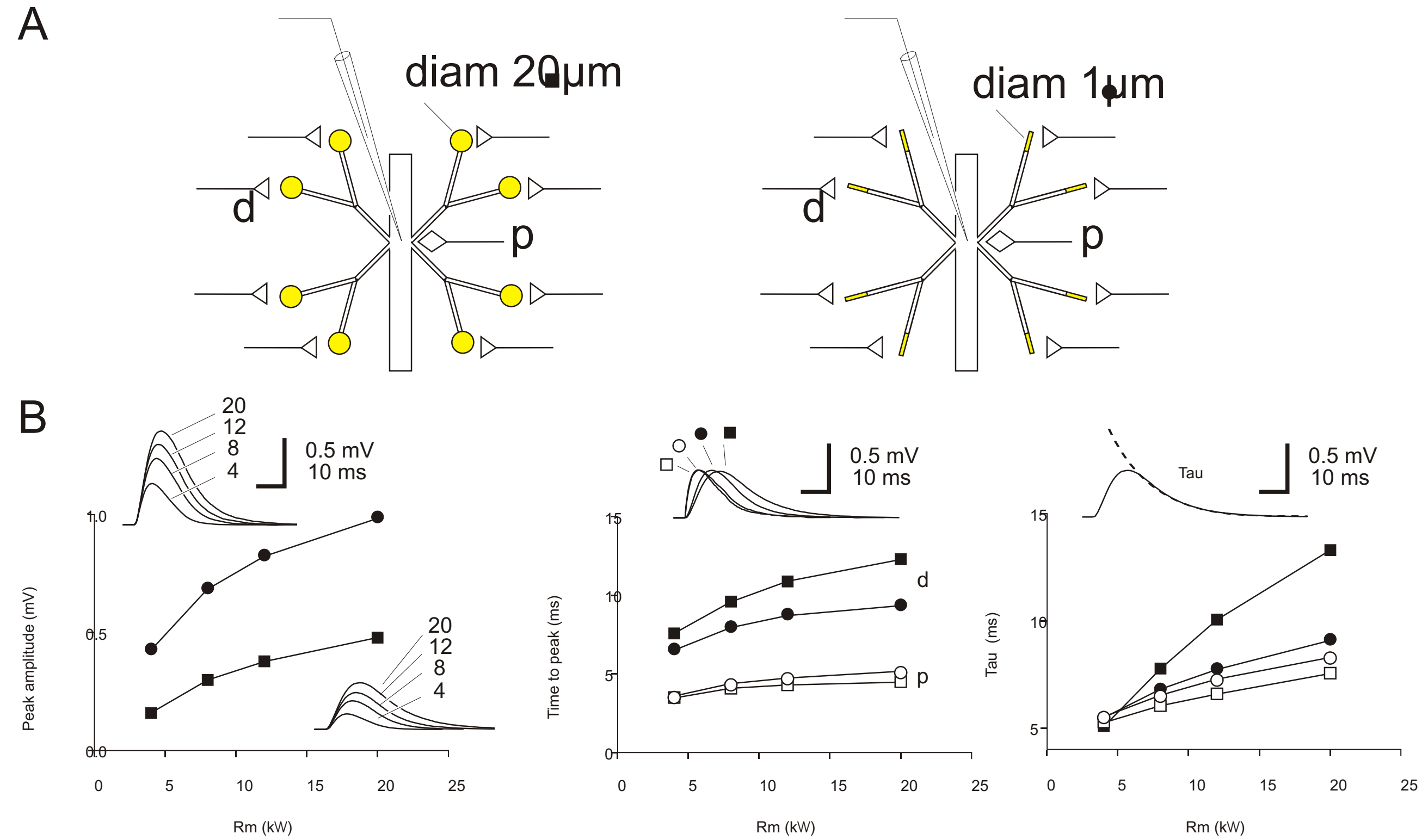

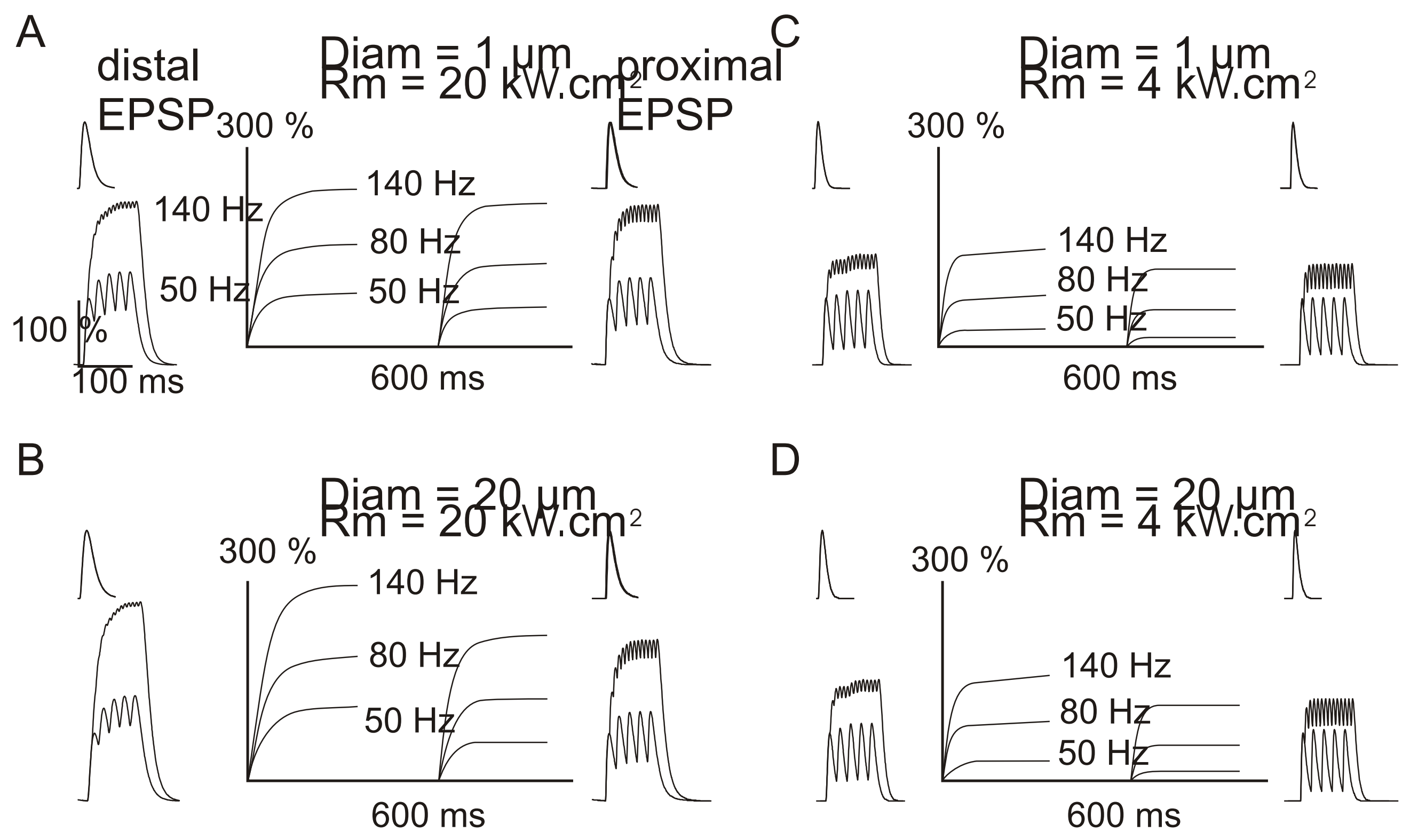